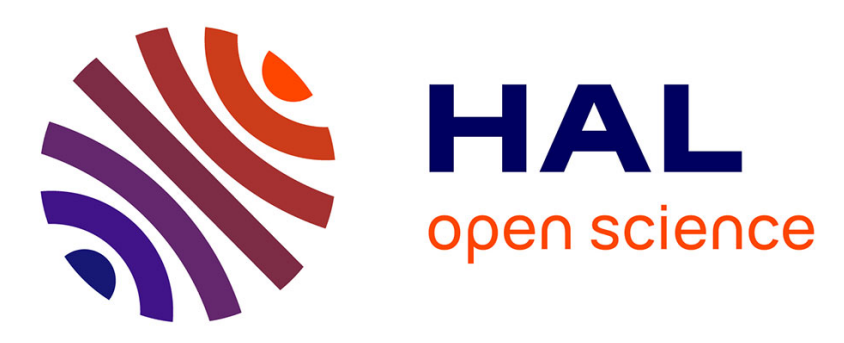

\title{
Fast In Vivo Imaging of SHG Nanoprobes with Multiphoton Light-Sheet Microscopy
}

Guy Malkinson, Pierre Mahou, Élodie Chaudan, Thierry Gacoin, Ali Sonay, Periklis Pantazis, Emmanuel Beaurepaire, Willy Supatto

\section{To cite this version:}

Guy Malkinson, Pierre Mahou, Élodie Chaudan, Thierry Gacoin, Ali Sonay, et al.. Fast In Vivo Imaging of SHG Nanoprobes with Multiphoton Light-Sheet Microscopy. ACS photonics, 2020, 7 (4), pp.1036-1049. 10.1021/acsphotonics.9b01749 . hal-02958634

\section{HAL Id: hal-02958634 https://hal.science/hal-02958634}

Submitted on 25 Nov 2020

HAL is a multi-disciplinary open access archive for the deposit and dissemination of scientific research documents, whether they are published or not. The documents may come from teaching and research institutions in France or abroad, or from public or private research centers.
L'archive ouverte pluridisciplinaire HAL, est destinée au dépôt et à la diffusion de documents scientifiques de niveau recherche, publiés ou non, émanant des établissements d'enseignement et de recherche français ou étrangers, des laboratoires publics ou privés. 


\section{Fast In Vivo Imaging of SHG Nanoprobes with Multiphoton Light- Sheet Microscopy}

Guy Malkinson, ${ }^{\perp}$ Pierre Mahou, ${ }^{\perp}$ Élodie Chaudan, Thierry Gacoin, Ali Y. Sonay, Periklis Pantazis, Emmanuel Beaurepaire,* and Willy Supatto*

Cite This: ACS Photonics 2020, 7, 1036-1049

Read Online

\section{ACCESS | Llll Metrics \& More | 回 Article Recommendations | (s) Supporting Information}
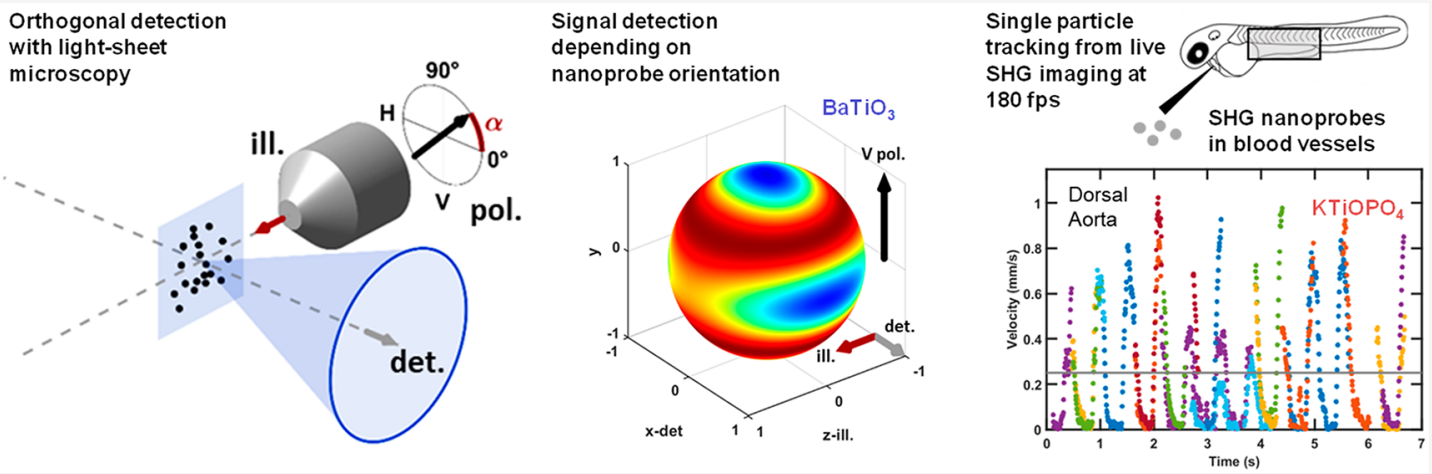

ABSTRACT: Two-photon light-sheet microscopy (2P-SPIM) provides a unique combination of advantages for fast and deep fluorescence imaging in live tissues. Detecting coherent signals such as second-harmonic generation (SHG) in 2P-SPIM in addition to fluorescence would open further imaging opportunities. However, light-sheet microscopy involves an orthogonal configuration of illumination and detection that questions the ability to detect coherent signals. Indeed, coherent scattering from micron-sized structures occurs predominantly along the illumination beam. By contrast, point-like sources such as SHG nanocrystals can efficiently scatter light in multiple directions and be detected using the orthogonal geometry of a light-sheet microscope. This study investigates the suitability of SHG light-sheet microscopy (SHG-SPIM) for fast imaging of SHG nanoprobes. Parameters that govern the detection efficiency of $\mathrm{KTiOPO}_{4}$ and $\mathrm{BaTiO}_{3}$ nanocrystals using SHG-SPIM are investigated theoretically and experimentally. The effects of incident polarization, detection numerical aperture, nanocrystal rotational motion, and second-order susceptibility tensor symmetries on the detectability of SHG nanoprobes in this specific geometry are clarified. Guidelines for optimizing SHGSPIM imaging are established, enabling fast in vivo light-sheet imaging combining SHG and two-photon excited fluorescence. Finally, microangiography was achieved in live zebrafish embryos by SHG imaging at up to 180 frames per second and single-particle tracking of SHG nanoprobes in the blood flow.

KEYWORDS: nanocrystal, nanoparticle, nonlinear microscopy, second-harmonic generation, single-plane illumination microscopy, zebrafish

Tight-sheet fluorescence microscopy (or single-plane illumination microscopy, SPIM) holds unique advantages for fast and three-dimensional (3D) in vivo imaging with low photodamage. $^{1-4}$ The introduction of multiphoton excitation in light-sheet microscopy has been shown to improve its imaging depth in scattering tissues. ${ }^{5,6}$ It also provided the opportunity to implement multimodal light-sheet imaging and combine two-photon excited fluorescence (2PEF) and other nonlinear contrast mechanisms, such as second-harmonic generation (SHG). ${ }^{5,7}$ However, detecting coherent signals in the orthogonal geometry of SPIM is not a trivial issue. Indeed, endogenous SHG signals obtained from, for example, fibrillar collagen, ${ }^{8}$ muscle fibers, ${ }^{9}$ or microtubules ${ }^{10}$ have been extensively studied in the context of point-scanning micros- copy (PSM), which uses a collinear configuration of illumination and detection. In this case, coherent signals from micron-sized structures have been shown to be directional and generated mostly in the forward direction, in the same direction as the excitation beam propagates. $8,11-15$ This directionality results from the coherent buildup of harmonic waves necessary for endogenous SHG signals to be

Received: December 11, 2019

Published: February 28, 2020 
detectable and from the large phase mismatch in all directions except for the propagation axis. Although this mechanism is expected to hamper largely orthogonal detection of such signals, the situation is quite different in the case of point sources such as SHG nanoprobes. ${ }^{16-18}$ Indeed, if a coherent source has dimensions smaller than the wavelength (Rayleigh regime assumption), efficient scattering may occur in multiple directions, ${ }^{19-22}$ although depending on the coupling between the incident laser polarization and the crystal properties. ${ }^{23}$

SHG nanoprobes have emerged as a promising tool for biological imaging applications. ${ }^{16-18}$ Several inorganic nanocrystals of noble metals or metal oxides with a noncentrosymmetric crystal structure have been used as nanometer SHG sources in nonlinear microscopy (or PSM). ${ }^{16,24,25}$ Unlike fluorophores, SHG nanoprobes are achromatic and can be excited at any wavelength. ${ }^{25-28}$ In addition, the SHG radiation spectrum is narrow when using femtosecond laser sources, which facilitates their selective detection. Since SHG is based on nonlinear scattering of light, no light absorption process is involved. Therefore, SHG nanoprobes usually have a high damage threshold, ${ }^{29,30}$ and the number of photons they radiate is not limited by saturation or photobleaching. ${ }^{31,32}$ Finally, nanostructures can efficiently scatter SHG in multiple directions. For example, signals from SHG nanoprobes using PSM geometry have been detected in the backward direction. ${ }^{32}$

Despite these unique optical properties, the use of SHG nanocrystals as probes in a light-sheet microscope, i.e., with orthogonal illumination and detection, has never been investigated. In this study, we theoretically and experimentally establish the use of $\mathrm{KTiOPO}_{4}(\mathrm{KTP})^{33,34}$ and $\mathrm{BaTiO}_{3}$ $(\mathrm{BT})^{32,35,36}$ nanocrystals as SHG nanoprobes for SHGSPIM. We analyze SHG signal collection efficiency as a function of microscope geometry (collinear in PSM vs orthogonal in SPIM), polarization direction, crystal structure, particle rotational motion, and detection numerical aperture. Specifically, we address the following questions: can signals from SHG nanoprobes be efficiently detected in the orthogonal geometry? Are the illumination intensities and signal levels in SHG-SPIM suitable for in vivo imaging? How do SHG signals from nanocrystals compare to endogenous structures with respect to orthogonal detection? What is the effect of the illumination polarization, and how should it be set to maximize signal mean and minimize its spread? What is the effect of nanocrystal rotational motion during imaging? Do the nature of the nanocrystal and of its susceptibility tensor symmetries matter?

\section{RESULTS}

Efficient Detection of SHG Nanoprobes Using the Orthogonal Geometry of Light-Sheet Microscopy. To select which SHG nanocrystals would be a good candidate for SPIM imaging, we considered the specificity of SPIM detection compared to PSM. Crystalline symmetries govern SHG radiation efficiency and directionality as a function of incident polarization. ${ }^{23}$ In PSM, the illumination polarization is generally linear and oriented in a plane that is orthogonal to the detection. By contrast, it should be realized that the illumination polarization can be oriented along the detection axis in SPIM. If the second-order susceptibility tensor $\chi^{(2)}$ of the SHG nanocrystal used has only diagonal elements $\left(\mathrm{d}_{11}, \mathrm{~d}_{22}\right.$, $\mathrm{d}_{33}$ as introduced in ref 23 and in the Methods), SHG will propagate orthogonally to the detection axis and will not be detected. As a consequence, we anticipated that nanocrystals with nondiagonal $\chi^{(2)}$ elements, therefore producing SHG propagating along the direction of the illumination polarization, would be more adapted to the orthogonal detection geometry of SPIM. In this study, we investigate this effect by comparing SHG-SPIM signals from nanocrystals with or without diagonal elements in their $\chi^{(2)}$. Among the 21 crystal classes that lack inversion symmetry and can produce SHG, none have purely diagonal elements. ${ }^{23}$ However, the diagonal elements can be significantly higher than the other nonzero elements, such as in the case of potassium titanyl phosphate, $\mathrm{KTiOPO}_{4}(\mathrm{~mm} 2$ class, biaxial orthorhombic crystal; see Methods). ${ }^{23,37}$ On the other hand, several crystals have lowvalue diagonal elements, such as barium titanate, $\mathrm{BaTiO}_{3}$ ( $4 \mathrm{~mm}$ class, uniaxial tetragonal crystal; see Methods). ${ }^{23,38}$ To investigate SHG signal detection in the orthogonal geometry of SPIM, we then chose to work with $\mathrm{KTP}^{33,34}$ and $\mathrm{BT}^{32,35}$ nanocrystals, which have been shown to be biocompatible and efficient SHG sources in point-scanning microscopy ${ }^{32,33}$ and should exhibit different results when imaged with SPIM.

To establish that SHG nanoprobes can be detected with SPIM, KTP and BT nanocrystals with a mean diameter of 150 $\pm 100 \mathrm{~nm}^{33}$ and $90 \pm 30 \mathrm{~nm},{ }^{35}$ respectively, were trapped in an agarose gel and mounted in a home-built multiphoton lightsheet microscope (Figure 1a,b). We recorded images with a linear incident polarization and experimental parameters similar to the ones previously used for biological 2P-SPIM imaging. ${ }^{39}$ Punctate signals were readily observed from the samples (Figure $1 \mathrm{~b}-\mathrm{d}$ ) with good signal-to-noise ratio (Figure $1 c, d)$. To confirm the nature of these signals, we confirmed that they exhibit SHG-specific properties. First, we verified that the intensity of the coherent signal generated by the nanocrystals was proportional to the square of the mean excitation power and did not saturate (Figure 1e,f). In addition, we confirmed that the signal spectrum was narrow and centered at half the excitation wavelength (Figure $1 \mathrm{~g}$ ). Finally, consistent with previous observations in PSM, ${ }^{34}$ we observed that the signal levels from individual nanocrystals exhibited large variations from one particle to another; this phenomenon can be explained by the sixth-power dependence of SHG with nanocrystal radius ${ }^{40}$ for subwavelength crystal sizes and by the various relative orientations between incident polarization and crystal axes ${ }^{41-43}$ (Figure $1 \mathrm{~h}$, and see below).

Since SHG signals generated by both nanocrystals and certain endogenous macromolecular assemblies such as fibrillar collagen, muscle fibers, or polarized microtubule bundles have been detected using multiphoton point-scanning microscopy, ${ }^{7-10,32}$ we compared the detection of SHG signals from nanocrystals and endogenous sources in the SPIM geometry to that in PSM. For that assay, we injected KTP nanocrystals to the trunk of zebrafish embryos, where skeletal muscles are an efficient source of endogenous SHG signals. ${ }^{32,44}$ We then measured the ratio of SHG signal levels between KTP and skeletal muscle in PSM and in SPIM (Figure S1). We found this ratio to be more than 1 order of magnitude stronger in SPIM than in PSM. This indicates that the orthogonal detection geometry does not allow efficient detection of endogenous SHG signals, while SHG nanoprobes are more efficiently detected. Together, these experiments show that, unlike in the case of endogenous sources of SHG, the SHG signals from nanocrystals can be efficiently detected in the orthogonal geometry using light-sheet microscopy. 

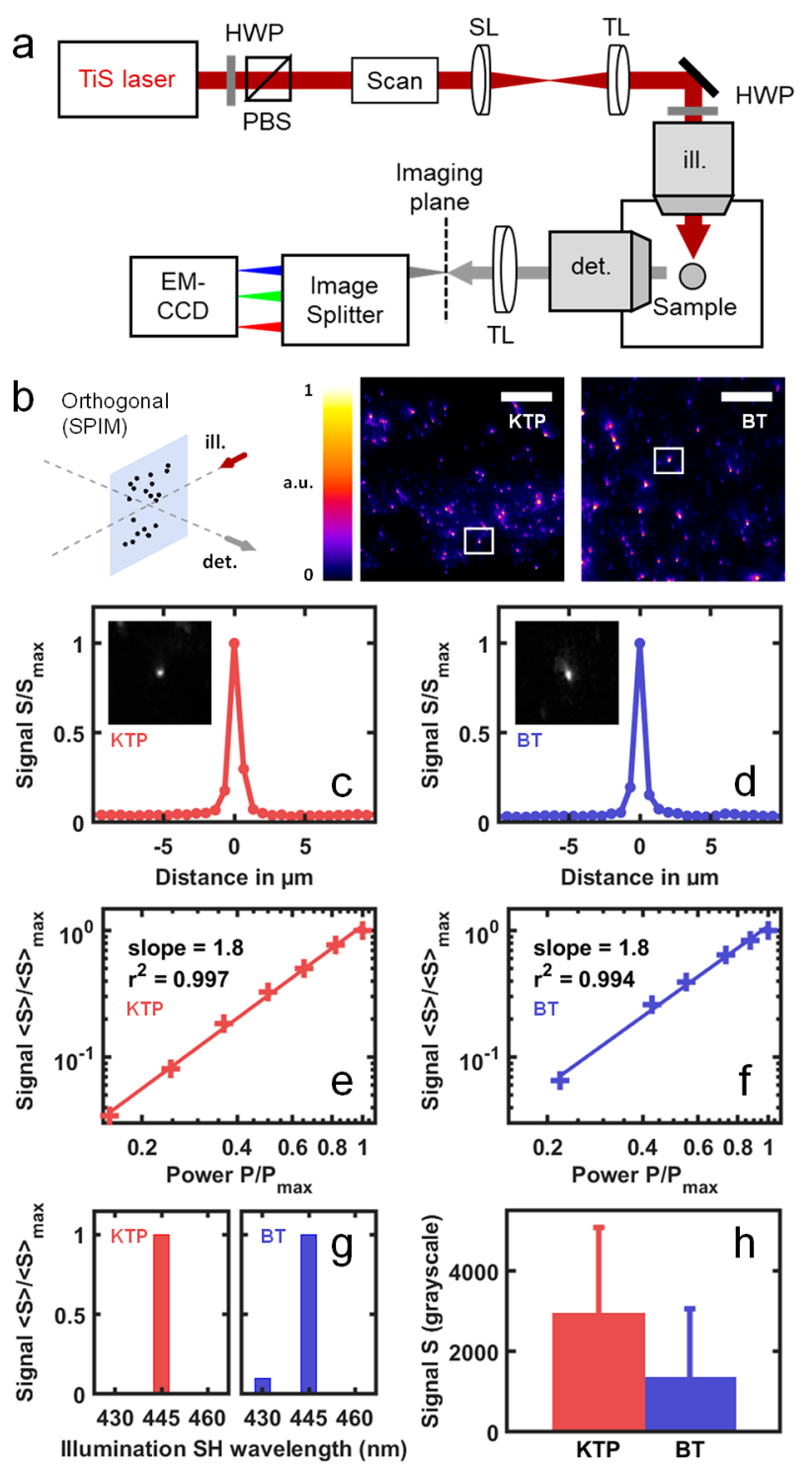

Figure 1. SHG signals from KTP and BT nanocrystals can be detected using the orthogonal geometry of a multiphoton light-sheet microscope. (a) Multiphoton light-sheet microscope (SPIM) setup. (b) SHG nanoprobes were mounted in agarose gel and imaged in orthogonal geometry (SPIM) at $\lambda=930 \mathrm{~nm}$. (c, d) Normalized SHG signal profiles depending on the horizontal distance to KTP (c, red) and BT (d, blue) nanocrystals selected from Figure $1 \mathrm{~b}$ images (insets in $c$ and $d$ and white boxes in b). (e, f) Quadratic dependence of normalized averaged SHG signal $\langle S\rangle /\langle S\rangle_{\max }$ on normalized illumination power $P / \mathrm{P}_{\max }$ is observed from both $\mathrm{KTP}$ (e, red) and BT (f, blue). (g) Spectral selectivity of SHG nanoprobes is observed by measuring the mean SHG signal detected using a $448 / 20$ bandpass detection filter depending on illumination wavelengths $(\lambda=860,890$, and $920 \mathrm{~nm}$ ). (h) Comparison of SHG signal levels obtained from KTP and BT nanocrystals in similar imaging conditions. HWP, half waveplate; SL, scan lens; TL, tube lens; PBS, polarization beam splitter; SH, second harmonic; ill., illumination; det., detection. Scale bar is $50 \mu \mathrm{m}$.

Application to Fast In Vivo Imaging of SHG Nanoprobes: From Microangiography to Multimodal Imaging in the Zebrafish Embryo. To demonstrate fast light-sheetbased SHG nanoprobe detection for biological imaging, we performed microangiography in zebrafish embryos. We seeded the blood flow within the dorsal aorta (DA) and the posterior cardinal vein (PCV) with SHG nanoprobes in 2 days postfertilization (dpf) embryos (see Methods). To measure the blood flow that is known to reach locally a $\sim 1 \mathrm{~mm} / \mathrm{s}$ velocity ${ }^{45}$ in zebrafish, we imaged individual SHG nanoprobes at 140 to 180 frames per second (fps) (Movie 1) flowing in the DA and the PCV of the embryo (Figure 2a,b and Movie 1). This frame rate corresponds to a $2-5 \mathrm{~ms}$ exposure time and up to $43 \mathrm{MHz}$ pixel rate. We confirmed that the instantaneous velocity measured from single nanoprobes exhibited pulsatile profiles due to the heart beating (Figure 2c) in the DA. ${ }^{45}$ By contrast, we detected a relatively steady flow with lower maximum velocities in the PCV (Figure 2c), as previously reported by tracking blood cells. ${ }^{45}$ The relatively small size of SHG nanoprobes compared to the blood vessel diameter enabled us to resolve the flow velocity profile across the vessel section, i.e., along the radial position $r$ (Figure 2a). To accomplish this, we extracted the maximum velocity of individual nanoprobes during a cardiac cycle depending on their radial position within the vessels (Figure 2d). We found that the radial flow profiles follow a parabolic curve, which is expected in the case of pulsatile flow with low Reynolds and Womersley numbers. ${ }^{46}$ Indeed, they are smaller than 1 since the measured heartbeat frequency is $<2 \mathrm{~Hz}$, the flow velocity is $<1 \mathrm{~mm} / \mathrm{s}$ (Figure 2c), the vessel diameter is smaller than 30 $\mu \mathrm{m}$, and the blood kinematic viscosity is $\sim 5 \times 10^{-6} \mathrm{~m}^{2} / \mathrm{s} .{ }^{45}$ Based on the radial flow profiles measured in the $\mathrm{DA}$ and $\mathrm{PCV}$, this fluid mechanics model of laminar flow predicted vessel diameters of 24 and $28 \mu \mathrm{m}$, respectively (Figure $2 \mathrm{~d}$ ). These predicted values are consistent with measurements obtained for the same embryonic stage using fluorescent labeling of the vessels. ${ }^{47}$ Finally, we performed multimodal and multicolor in vivo imaging by combining the detection of green and red twophoton excited fluorescence with SHG from nanoprobes. A 2.5 dpf zebrafish embryo expressing eGFP in its endothelial cells and dsRed in its red blood cells was injected with KTP nanocrystals and imaged at $47 \mathrm{fps}$ using a single excitation wavelength $(930 \mathrm{~nm})$ with a multichannel spectral detection (Figure S2 and Movie 2). Since SHG nanoprobes are achromatic, multimodal imaging should be possible at any excitation wavelength and the most adapted to the fluorophore absorption can be selected. To illustrate this advantage, we used $1060 \mathrm{~nm}$ excitation to image the red fluorescent protein mCherry simultaneously with KTP nanoprobes seeding the blood flow in the embryo's brain at $65 \mathrm{fps}$ (Movie 3). Together, our results demonstrate that fast light-sheet imaging of SHG nanoprobes can be used as a reliable technique to resolve blood flow dynamics at micrometer scales in vivo. Moreover, it can be combined straightforwardly with twophoton excited fluorescence to perform multimodal and multicolor in vivo imaging.

No Toxicity or Photodamage Observed after Injection of SHG Nanoprobes and during Fast In Vivo Imaging. To test whether the injection of KTP or BT nanocrystals into the zebrafish embryo circulation disrupts normal biological processes, we monitored the embryo development after injection performed at 2 or $3 \mathrm{dpf}$. Several physiological parameters were examined: developmental stages, overall morphology, swimming, and response to touch. We did not observe any abnormalities in injected embryos up to $5 \mathrm{dpf}(N=30$ embryos; not shown). Likewise, KTP nanocrystal injection to fertilized zebrafish eggs did not result in developmental disruptions $(N=10$ eggs; not shown), confirming previous reports in the case of $\mathrm{BT}$ nanocrystals. ${ }^{32}$ 

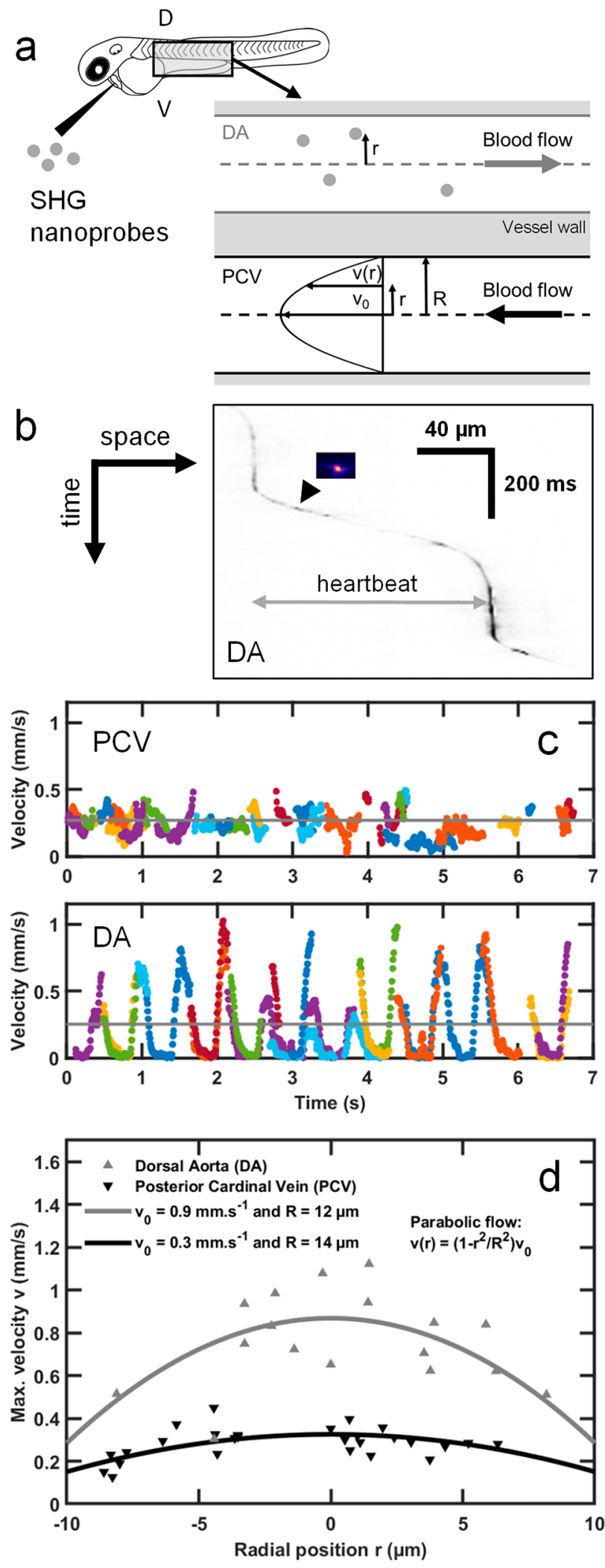

Figure 2. Microangiography in zebrafish embryos using fast in vivo SHG imaging and tracking of individual SHG nanoprobes seeding the blood flow. (a) KTP nanocrystals were injected in the blood vessels of 2 dpf zebrafish embryos and were imaged in both the dorsal aorta (DA) and the posterior cardinal vein (PCV). Single nanoprobes were tracked along the blood vessel and trajectories were constructed for each particle. (b) Kymogram (space-time graph with inverted grayscale signals) of representative tracked SHG nanoprobe (inset

Figure 2. continued

image) seeding the dorsal aorta and exhibiting a pulsatile movement. (c) Cumulative plot of KTP nanocrystal speeds during 12 heartbeats. Nineteen and 37 nanocrystals flowing in the DA and the PCV, respectively, were imaged in vivo at $140 \mathrm{fps}$ and tracked. Their instantaneous velocities are drawn as a function of time. Different trajectories (in different colors) were used to construct the plotted profiles. The pulsatile nature of their movement in the DA, owing to the systole/diastole cycles, enables extracting the heartbeat frequency, which is $\sim 2 \mathrm{~Hz}$. The mean velocity is indicated with gray lines $(250 \pm$ 80 and $270 \pm 20 \mu \mathrm{m} / \mathrm{s}$, for DA and PCV, respectively). (d) Microangiography and in vivo tracking of individual SHG nanoprobes flowing in blood vessels demonstrates different velocities along the vessel's diameter. Shown are two plots taken from the DA (gray, uppointing triangles) and from the PCV (black, down-pointing triangle). The trajectories were drawn with respect to the position $r$ of the trajectory along the diameter of the blood vessel (a), where the center was estimated as the average of the two most peripheral trajectories in the respective blood vessel. In general, particles were found to maintain their relative position along the vessel, with respect to their initial positions. The plots show that particles that flow in the center exhibit an overall higher range of flow velocities (max. velocities correspond to the average of the $5 \%$ fastest time points during a track) compared to particles that are close to the vessel walls, as expected from a parabolic flow (gray and black lines, for artery and vein, respectively, represent parabolic fits).

To investigate potential photodamage induced during in vivo imaging, we systematically monitored the heartbeat frequency of the live embryos during SPIM imaging sessions by directly imaging the heart or by extracting its beating frequency from the blood flow pattern as shown above (Figure $2 \mathrm{~b}$ ). We found that even under the strongest illumination $(150 \mathrm{~mW}$ and $\sim 0.1$ focusing numerical aperture, corresponding to $85 \mathrm{MW} / \mathrm{cm}^{2}$ peak irradiance, which is 2 orders of magnitude below the previously reported nonlinear photodamage threshold ${ }^{48}$ ), the heart was beating at frequencies within the normal range for $2-3$ dpf embryos ${ }^{45,49}$ (typically $\sim 2 \mathrm{~Hz}$ at room temperature, $N$ $=5)$. In addition, we investigated photobleaching of fluorescent proteins and photostability of SHG nanoprobes during multimodal imaging. We monitored GFP and KTP signals during high-speed in vivo SPIM imaging of blood vessels in fli1-eGFP transgenic embryos ${ }^{50}$ that were injected with KTP nanocrystals. We simultaneously imaged eGFP and KTP nanocrystals at $\sim 70 \mathrm{fps}, 930 \mathrm{~nm}$ illumination wavelength, and $150 \mathrm{~mW}$ mean power. Both $2 \mathrm{PEF}$ and SHG signals remained constant (within $<5 \%$ variation in the case of $2 \mathrm{PEF}$ ) after 2000 frames, corresponding to $30 \mathrm{~s}$ of constant imaging (Figure S2). Finally, we monitored the development of the embryos used in this study during the days following injection of KTP or BT nanocrystals and imaging with SPIM. We observed the physiological parameters listed above and noted that they stayed within normal ranges up to $5 \mathrm{dpf}(N=10$ embryos; not shown). Together, these results confirm that no significant photobleaching of fluorescent protein or photodamage by SHG nanoprobes was observed during in vivo image acquisition and that embryos injected with SHG nanoprobes and imaged with light-sheet microscopy developed normally.

SHG Signal Is Less Sensitive to Incident Polarization in the Case of BT than in the Case of KTP Nanocrystals. The large fluctuation of SHG signals we observed when imaging individual KTP nanocrystals in vivo made singleparticle tracking difficult (Movie 1). To optimize SPIM 

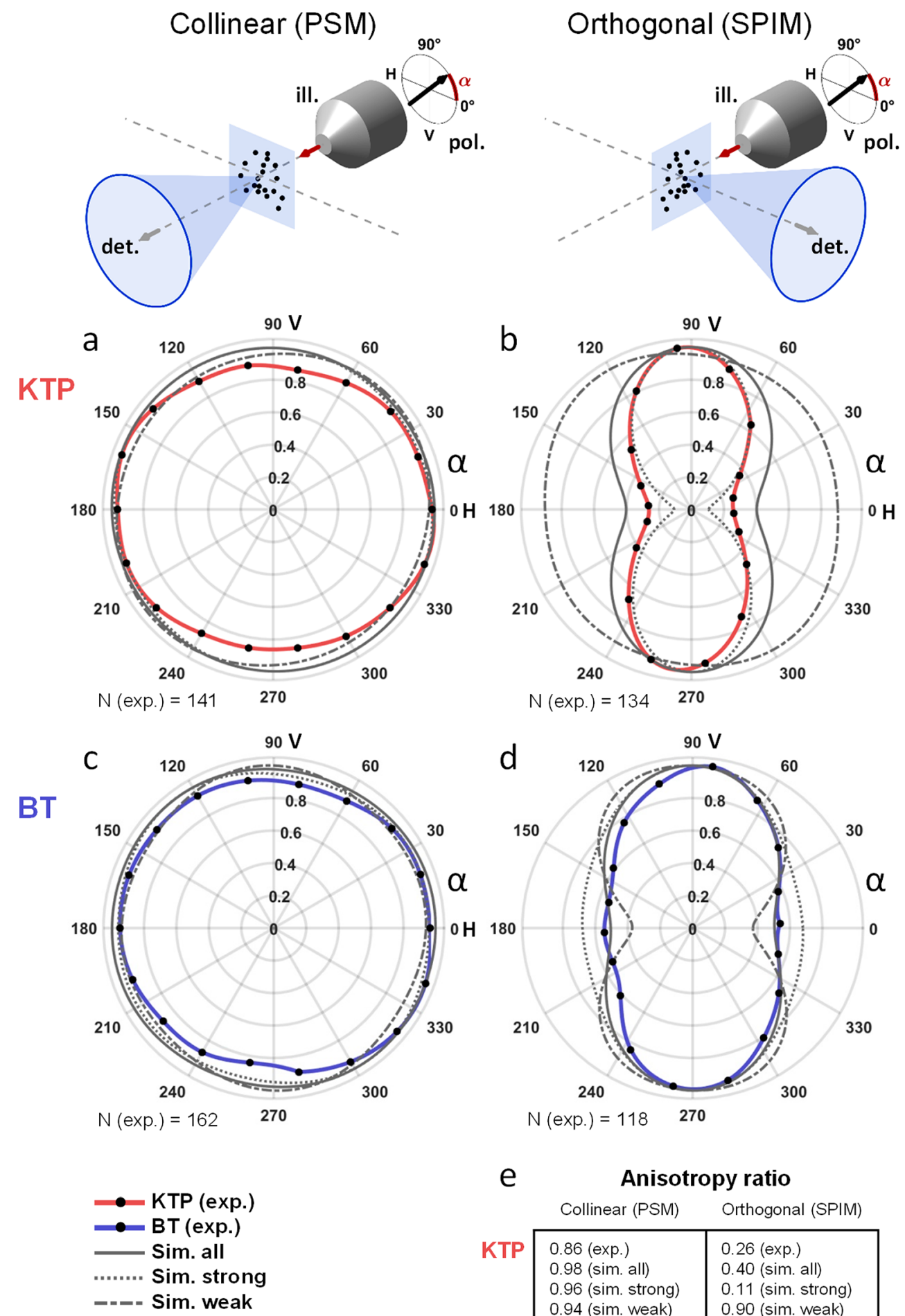

\begin{tabular}{cl|l|}
\multicolumn{1}{c}{ e } & \multicolumn{2}{c}{ Anisotropy ratio } \\
\multicolumn{1}{c}{} & \multicolumn{1}{c}{ Collinear (PSM) } & \multicolumn{1}{c}{ Orthogonal (SPIM) } \\
\cline { 2 - 3 } KTP & 0.86 (exp.) & 0.26 (exp.) \\
& 0.98 (sim. all) & 0.40 (sim. all) \\
& 0.96 (sim. strong) & 0.11 (sim. strong) \\
& 0.94 (sim. weak) & 0.90 (sim. weak) \\
\cline { 2 - 3 } BT & 0.84 (exp.) & 0.53 (exp.) \\
& 0.97 (sim. all) & 0.50 (sim. all) \\
0.94 (sim. strong) & 0.68 (sim. strong) \\
0.92 (sim. weak) & 0.37 (sim. weak) \\
\hline
\end{tabular}

Figure 3. Mean detected SHG signals strongly depend on illumination polarization orientation in light-sheet microscopy. (a-d) Mean SHG signal detected (from $N=118$ to 162 nanoprobes) as a function of the illumination linear polarization angle (black dots) for KTP (a and b) or BT (c and d) nanocrystals (spline interpolation in red and blue lines, respectively). Experiments were conducted using either a collinear (PSM, a and c) or an orthogonal (SPIM, b and d) geometry. Results of simulation (1000 SHG nanoprobes with random nanocrystal orientations) are indicated with plain gray lines, and signal means from $20 \%$ strongest or weakest nanocrystals are plotted with dotted or dashed gray line, respectively. Angles of $0^{\circ}$ and $90^{\circ}$ corresponds to horizontal $(\mathrm{H})$ and vertical $(\mathrm{V})$ polarizations that are oriented along and orthogonal to the detection axis in SPIM, respectively. (e) The anisotropy ratio corresponds to the minimum-to-maximum signal from results plotted in (a) -(d). ill., illumination; det., detection; sim., simulation; exp., experiment; V, vertical; $\mathrm{H}$, horizontal, $\mathrm{N}$, number of experimental nanocrystals used. 
imaging of SHG nanoprobes for such biological application, we investigated whether these fluctuations could be due to the crystal nature and compared KTP and BT crystals. Since the nonlinear signal from SHG nanoprobes is known to depend on the incident polarization in $\mathrm{PSM}^{43}$ we first explored the impact of linear polarization on average detected SHG signals depending on the nanocrystal nature and the microscope geometry. We performed numerical calculations of SHG signal detected as a function of incident polarization for 1000 simulated and randomly oriented nanocrystals of both KTP and BT types. The theory in this purpose is detailed in the Methods section. We then experimentally validated the results from numerical simulations by imaging KTP and BT nanocrystals trapped in agarose gel.

First, simulations in PSM confirmed that the average signal detected from randomly oriented SHG nanoprobes did not depend on the orientation of the linear polarization (plain gray lines in Figure 3a and $c$ ), even if the signal from individual nanocrystals was polarization-dependent. For both KTP and BT nanocrystals, the simulated anisotropy ratio was very close to 1 (Figure $3 \mathrm{e}$ ). This result was confirmed experimentally by imaging 141 and $162 \mathrm{KTP}$ and BT nanocrystals, respectively (red and blue lines in Figure $3 \mathrm{a}$ and c, anisotropy ratio $>0.84$ ). We note that the experimental design in PSM dictates that the linear polarization remains always oriented orthogonally to the detection axis, which explains this observation.

By contrast, the situation was much different in the orthogonal detection geometry of SPIM, as shown in Figure $3 \mathrm{~b}$ and $\mathrm{d}$. The average SHG signal from simulated nanoprobes was consistently strongest when using vertical $(\mathrm{V})$ polarization and weakest when using horizontal $(\mathrm{H})$ polarization, corresponding to a polarization orthogonal or parallel to the SPIM detection axis, respectively (plain gray lines in Figure $3 \mathrm{~b}$ and d). In agreement with simulations, the average SHG signal from nanocrystals imaged in a gel was drastically reduced when the polarization was oriented along the SPIM detection axis (red and blue lines in Figure $3 \mathrm{~b}$ and $\mathrm{d}$, respectively). Interestingly, both simulations and experiments show that, in SPIM, SHG signals from BT nanocrystals exhibited a weaker dependence on polarization than in the case of KTP nanocrystals (Figure $3 \mathrm{~b}$ and $\mathrm{d}$ ). Indeed, the anisotropy ratio measured experimentally was 2-fold lower in the latter case ( 0.26 and 0.53 , for KTP and BT nanocrystals, respectively). We note that this ratio is larger than the depolarization ratio obtained with hyper-Rayleigh scattering ( 0.36 for BT nanocrystals in ref 21 , for instance) since SPIM imaging uses a significantly larger detection aperture.

Together, these data demonstrate that, unlike in PSM, average signal intensities from SHG nanoprobes in SPIM exhibit a large dependence on the orientation of the linear polarization of the illumination. The average SHG signal is significantly reduced when the polarization is oriented along the SPIM detection axis and is maximized when the polarization is oriented orthogonally to the detection axis. In addition, this polarization sensitivity depends on the nature of the nanocrystal. Indeed, signals from KTP nanocrystals exhibited a stronger sensitivity to the incident polarization than BT signals. More precisely, the KTP nanocrystals with the strongest detected signals exhibited the strongest sensitivity to incident polarization (see Supporting Results 1). By contrast, the BT nanocrystals with the strongest detected signals were weakly sensitive to incident polarization. As we anticipated when choosing to compare KTP and BT nanocrystals, this phenomenon can be explained by the structure of their $\chi^{(2)}$ tensors. Since these results have important practical consequences for imaging applications, we further investigated how to optimize the detection of SHG nanoprobes in the SPIM geometry.

Polarization Orientation Modulates Both Distribution Mean and Spread of SHG-SPIM Signals. To gain insights into the polarization sensitivity and the effect of nanocrystal orientation, we analyzed the distribution of simulated SHG signal levels from randomly oriented nanocrystals depending on the crystal nature and the microscope geometry (Figure 4a,b). To compare our results with previous observations on BT nanocrystals in PSM, ${ }^{43}$ we used the same metric and estimated the relative standard deviation of the detected SHG signals (i.e., the standard deviation divided by the mean) to quantify the spread around the mean.

As expected, we observed no difference in the signal distribution between $\mathrm{V}$ - and $\mathrm{H}$-polarizations in the case of PSM (Figure 4a, in red for KTP and in blue for BT, vertical and horizontal arrows). By contrast, the difference was pronounced in the case of SPIM orthogonal geometry (Figure $4 \mathrm{~b}$, in red for KTP and in blue for BT, vertical and horizontal arrows). Indeed, when the linear polarization was orthogonal to the detection axis, the SHG signal distribution was similar both in PSM and in SPIM (V-polarization in Figure 4a,b). However, in the case of a linear polarization parallel to the detection axis in SPIM, the resulting SHG signal distribution was significantly different in mean and spread (H-polarization in Figure $4 a, b)$ : the signal mean was $40 \%$ and $51 \%$ that of $\mathrm{V}$ polarization for KTP and BT, respectively. Interestingly, this difference depends on the nanocrystal nature. While for both nanocrystals the signal mean decreased when using an $\mathrm{H}$ compared to a V-polarization, the relative standard deviation decreased in the case of KTP (from $94 \%$ to $47 \%$, Figure $4 \mathrm{~b}$ ) and increased in the case of BT (from $36 \%$ to $51 \%$, Figure $4 \mathrm{~b}$ ). This implies that the use of a V-polarization in SPIM optimizes the signal mean level. However, its consequences on signal spread depend on the nanocrystal nature: while it significantly increased the signal spread in the case of KTP nanocrystals, it reduced this spread in the case of BT nanocrystals. These simulations explained the large fluctuation of SHG signals observed when tracking KTP nanoprobes experiencing rotational motion when seeding the blood flow (Figure 2 and Movie 1). They also indicated that the mean and spread of SHG recorded from nanoprobes of different orientations depend on the detection NA in different manners for KTP and BT nanocrystals (Figure S3 and Supporting Results 2).

Circularly Polarized Light Is Not Advantageous for SPIM Imaging of SHG Nanoprobes. In PSM for biological SHG imaging, circularly polarized light was shown to mitigate the spread of SHG signal intensities and equally excite all harmonophore orientations ${ }^{8,51}$ even at the cost of reducing the average signal. In the case of BT nanocrystals imaged with PSM, it has been shown that the benefit of circular polarization is lost, since it reduces the average signal without reducing the relative standard deviation. ${ }^{43}$ We reproduced this last result on BT nanocrystals with our simulations (Figure $4 \mathrm{a}$ in blue). We found however that circular polarization can still be a benefit for imaging KTP nanocrystals in PSM since it significantly reduces the relative standard deviation of the signals (from $94 \%$ to $61 \%$, Figure $4 \mathrm{a}$ in red). By contrast, we did not find any benefit of using a circularly polarized light in SPIM. Indeed, a stronger average signal with a lower signal spread could still be 


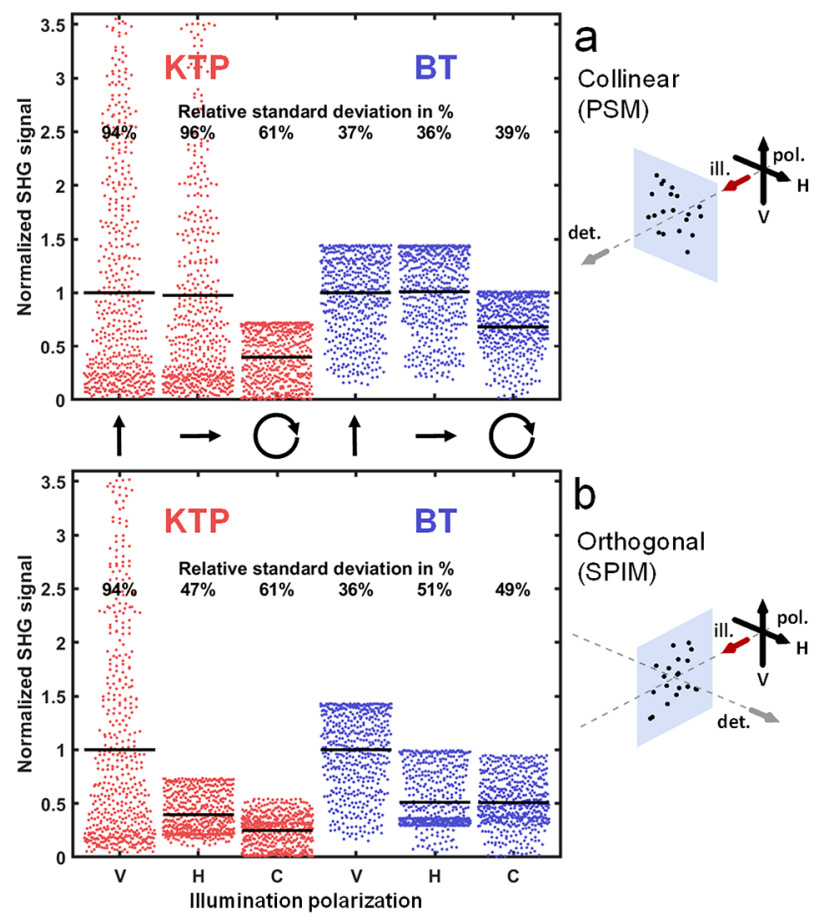

Figure 4. continued

mean (black horizontal lines). Signal for each polarization is normalized to the vertical polarization case. Signal mean and relative standard deviation are indicated for 10000 nanocrystals simulated with random orientations. $(\mathrm{c}-\mathrm{g})$ Signal intensity distribution for all possible nanocrystal orientations for KTP (d, e) and BT (f, g) nanocrystals using vertical ( $d$ and $f$ ) or horizontal (e and $g$ ) linear polarization in SPIM with a 0.8 detection NA. Results are represented on a unit sphere, where the position on the sphere corresponds to the nanocrystal orientation relative to the microscope reference frame as shown in (c), and color corresponds to the signal intensity detected. Red, gray, and black arrows correspond to the SPIM illumination axis, detection axis, and linear polarization orientation, respectively. Represented signal is a spline interpolation of signals from 4000 nanocrystals simulated with random orientations. The signal level is normalized to the maximum value in each case. ill., illumination; det., detection; $\mathrm{V}$, vertical; $\mathrm{H}$, horizontal.

obtained either when KTP nanocrystals were imaged with an $\mathrm{H}$-polarization (Figure $4 \mathrm{~b}$ in red) or when BT was imaged with a V-polarization (Figure $4 \mathrm{~b}$ in blue). Altogether, these data show that the strategy of using a circularly polarized illumination is inefficient in SPIM imaging of SHG nanoprobes and that a linear polarization should be used instead.

The Nature of the Nanocrystals Plays a Critical Role

C
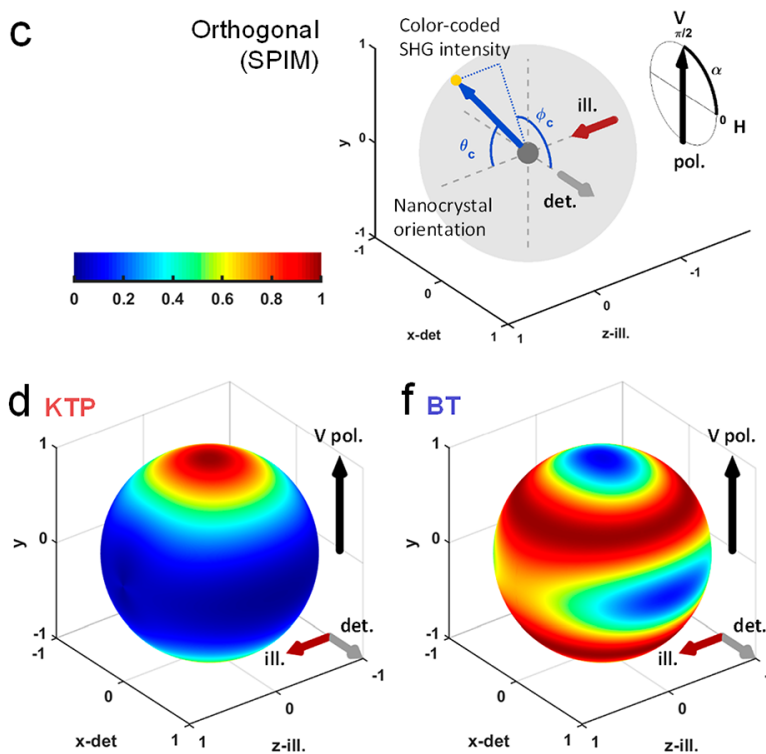

f $\mathrm{BT}$

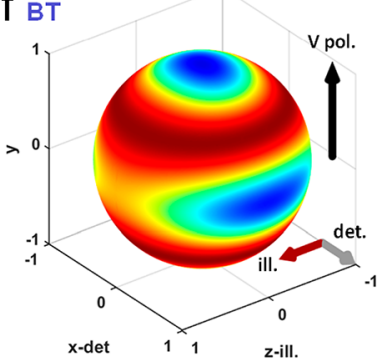

e KTP
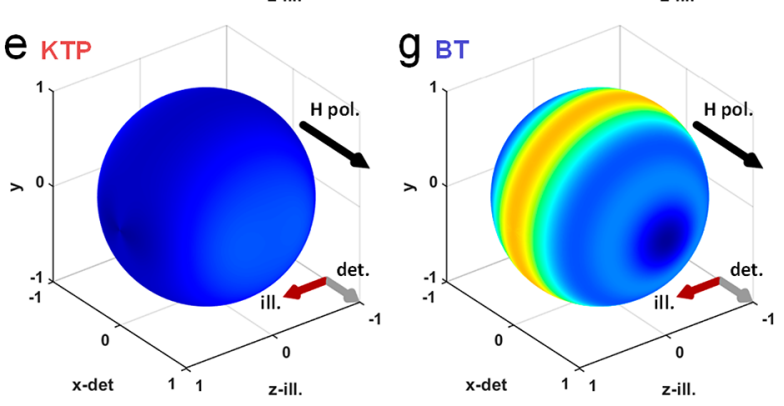

Figure 4. Detected SHG signal distribution depending on nanocrystal orientation and illumination polarization: theoretical investigation. (a, b) Simulation of SHG signal distributions from $500 \mathrm{KTP}$ (red) and BT (blue) nanocrystals using linear vertical (V), linear horizontal $(\mathrm{H})$, or circular $(\mathrm{C})$ polarization of the illumination light in the collinear ( $\mathrm{a}$, PSM) or orthogonal (b, SPIM) geometry, using a 0.8 detection numerical aperture (NA). Random distribution of nanocrystal orientation results in spreading of signal levels around the in SPIM Imaging of SHG Nanoprobes. To confirm that the relative values of the nanocrystal $\chi^{(2)}$ tensor elements are critical for efficient SHG-SPIM imaging, we simulated the detected signal intensity for all possible crystal orientations and investigated the effect of crystal nature and incident polarization (Figure $4 c-g$ and Figure S4). Simulated data are represented on a unit sphere, where the position on the sphere corresponded to the $3 \mathrm{D}$ crystal orientation and the color corresponded to the signal intensity detected as shown in Figure $4 \mathrm{c}$. Figure $4 \mathrm{~d}-\mathrm{g}$ show the signal intensity detected in the orthogonal direction with a 0.8 numerical aperture objective in the KTP and BT cases, with V- or H-polarization. The detected signal was then decomposed into the total generated SHG (Figure S4a-d) and the SHG collection efficiency (Figure $\mathrm{S} 4 \mathrm{e}-\mathrm{h}$ ). Simulations show that KTP nanocrystals behave as simple dipoles, resulting in a straightforward scattering pattern. As a result, a large signal can be obtained only when the KTP main axis is aligned with the incident polarization (Figure $4 \mathrm{~d}$ ). This behavior is described by the low value of nondiagonal elements in the $\mathrm{KTP} \chi^{(2)}$ tensor (see Methods) and is characterized by an absence of propagation along the direction of the incident polarization. It also revealed why for a uniform distribution of nanocrystal orientations the distribution of signal levels was shifted toward weak signals as observed in Figure $4 \mathrm{~b}$ (red, Vpolarization). When the illumination polarization was rotated toward the detection axis, the total SHG pattern rotated accordingly (Figure S4a,b). However, SHG was mainly generated in directions orthogonal to the detection axis and was poorly collected (Figure S4f). As a result, the detected signal was weak for all nanocrystal orientations (Figure 4e), explaining the observed signal distribution (Figure 4b, red, $\mathrm{H}$ polarization). By contrast, BT was capable of polarization conversion during the SHG process, which was characterized by the presence of nondiagonal elements in its $\chi^{(2)}$ tensor $^{38}$ (see Methods). As a consequence, more scattering directions were allowed than in the case of KTP, and only a limited number of crystal orientations resulted in a weak detected 


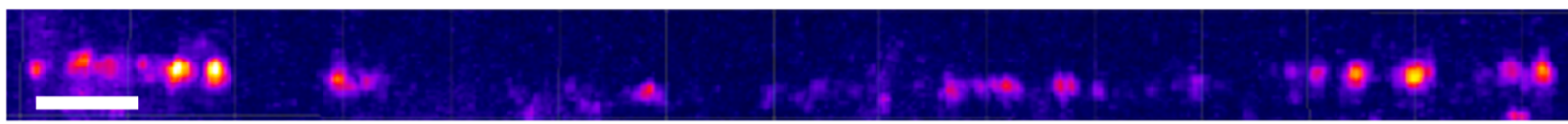

KTP

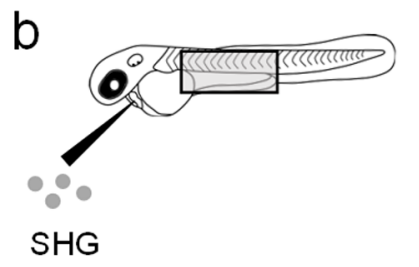

nanoprobes

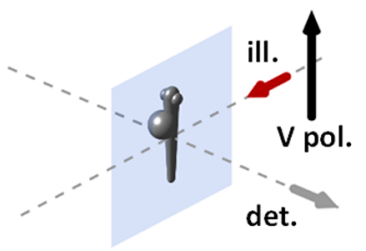

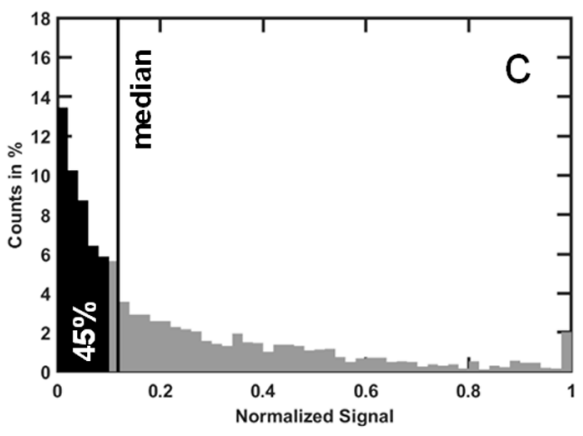

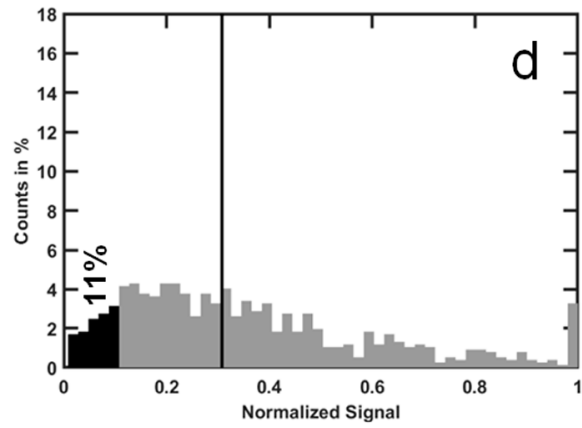

Exp.
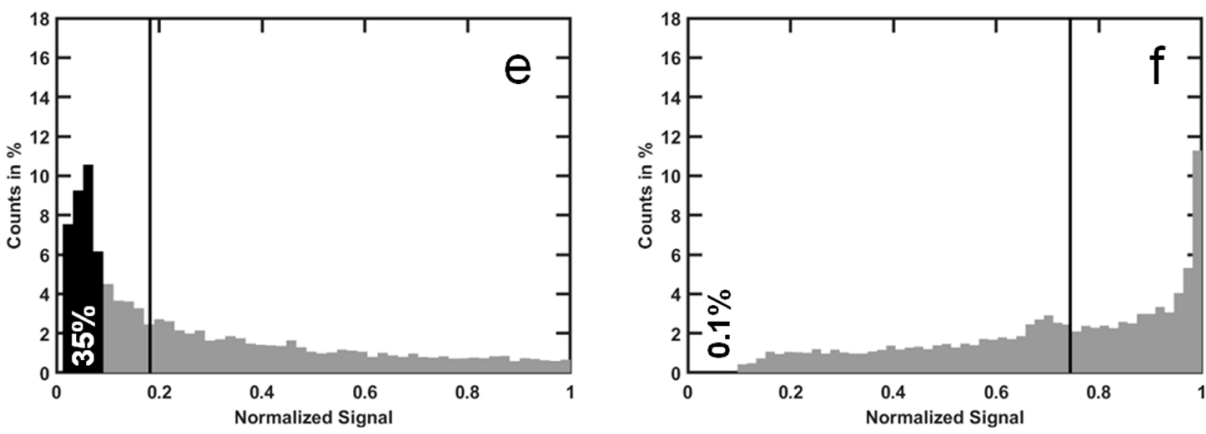

Sim. 1

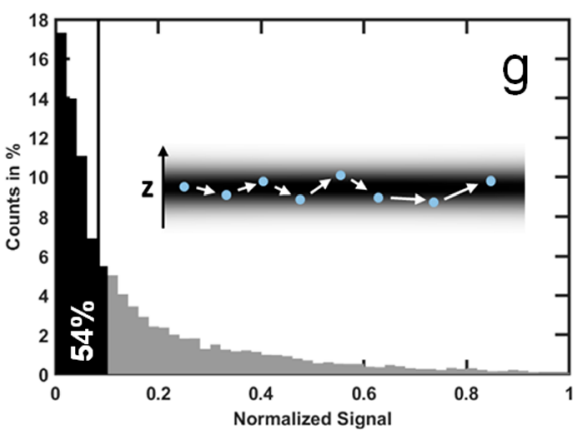

Sim. 2

Figure 5. Optimizing SHG signals detected from individual SHG nanoprobes experiencing rotational motion: experimental investigation. Analysis of SHG nanoprobe signal recorded in vivo shows that BT and KTP nanocrystals generate different patterns of dynamic ranges as they flow. ( $a, b)$ A solution containing SHG nanoprobes was injected to a zebrafish embryo and imaged at $140 \mathrm{fps}$ and $5 \mathrm{~ms}$ exposure time using SPIM with vertical linear polarization of the excitation beam. Single nanoprobes were tracked off-line. Shown in (a) is a maximal projection of selected time points where the different locations of a single BT nanocrystal in the blood vessel of a zebrafish embryo can be seen (scale bar $20 \mu \mathrm{m}$ ). (c, d) Histograms depicting the signal levels of KTP (c) and BT (d) nanocrystals that were tracked in data sets such as the one presented in (a). For each of the particles that were tracked off-line, a trajectory of the particle's path was constructed, and the intensities in the different positions were then normalized to the maximal value along that trajectory. The histograms depict the pooled data from all trajectories per particle type. Note that the BT histogram is more skewed to the right-hand side with respect to the KTP histogram, suggesting that while a BT nanocrystal is flowing and rotating, its range of generated SHG intensities is closer to the maximal intensity, compared to KTP. (e, f) Simulated histograms (Sim. 1) corresponding to the previous experimental cases $(c, d)$ confirming the difference between KTP and BT nanocrystals: the histogram is skewed to low intensities in the case of KTP (e) and to high intensities in the case of BT (f). (g, h) By adding the random positioning of the nanocrystals through the $z$-thickness of the light-sheet to the simulation (Sim. 2), the resulting histograms are similar to experimental ones. sim., simulation; exp., experiment.

signal (Figure 4f). Such behavior explains the observed signal distribution that is shifted toward large signals (Figure $4 \mathrm{~b}$, blue, V-polarization). Altogether, these results revealed how the structure of the crystal $\chi^{(2)}$ tensor is responsible for the differences shown in Figures 3, 4, and S4 between KTP and BT nanocrystals. More generally, they demonstrate that the nature of the nanocrystals has a critical impact on the detected SHG signal distribution in light-sheet microscopy.
BT Is More Adapted than KTP for In Vivo Particle Tracking Using SHG-SPIM: An Experimental Validation. The strong dependence of detected SHG signal intensity on nanocrystal relative orientation has an important consequence for applications such as particle tracking using SHG-SPIM. Indeed, a tracked SHG nanoprobe will seemingly "blink" as it undergoes rotational motion during its movement (Figure $2 \mathrm{~b}$, Movie 1). According to the above analyses, this effect should be more pronounced in the case of KTP than in the case of BT 
nanocrystals. To validate this prediction with in vivo experiments, we imaged SHG nanoprobes injected in the blood circulation of a zebrafish embryo with SPIM at typically 90 to $180 \mathrm{fps}$ and 1 to $5 \mathrm{~ms}$ exposure time using a V-polarization (Figure 5a,b and Movies 1 and 4, for KTP and BT cases, respectively). We then tracked SHG nanoprobes and computed the histograms of normalized signal levels recorded along nanoprobe trajectories (Figure 5c,d and see Methods for details) and compared them with simulations from randomly oriented nanocrystals (Figure 5e,f). As expected from our simulations (Figure $4 \mathrm{~b}$ ), the histograms from simulated KTP and BT nanocrystals exhibited opposite shapes with a shift toward weak signals in the case of KTP (35\% of nanoprobes generating a signal below $10 \%$ of the maximum, Figure $5 \mathrm{e}$ ) and toward large signals in the case of BT $(0.1 \%$ of nanoprobes generating a signal below $10 \%$ of the maximum, Figure 5f). While this difference was also significant in experiments, it was not as pronounced as in our first simulation (45\% and $11 \%$ of generated signals below $10 \%$ of the maximum for KTP and BT, respectively, in Figure 5c,d). To better match the simulations with the experimental data, we took into account the movements of nanoprobes in and out of the light-sheet along each trajectory (Figure 5g,h and Methods). Altogether, these results demonstrate fast rotational motion of SHG nanoprobes circulating in a biological flow in vivo can result in large fluctuations of signal levels, which can hamper particle tracking. To mitigate this effect, the nature of the SHG nanocrystal is critical and BT is more adapted than KTP for this purpose.

\section{DISCUSSION}

Detection of Extended and Point Sources of SHG in SPIM. In this study, we report on the inefficient detection of endogenous SHG using the orthogonal geometry of SPIM compared to PSM. Indeed, we show that SHG signals from skeletal muscle in the zebrafish trunk are barely above the background level when using SPIM under standard imaging conditions. Essentially, detectable SHG signals arise from endogenous spatially extended sources, which results in a strong directionality of the SHG radiation in the collinear direction and a poor detection in the orthogonal geometry of SPIM. For this reason, the size of the crystal used for SHGSPIM is critical: to behave as a point source and to radiate SHG signals in all directions of space, the crystals have to be significantly smaller than the illumination wavelength.

Guide through Parameters Governing SHG Signal Detection in SPIM Using Nanocrystals. Here, we analyzed the parameters governing signal detection level and distribution when using SHG nanoprobes in SPIM, namely, the polarization of the incident light, the nature and orientation of the nanocrystals, and the detection aperture. We highlighted the difference between SPIM and PSM detection geometries. For instance, the ability to image SHG nanoprobes with a linear polarization that is parallel to the detection is specific to SPIM and has important consequences. Our analysis provides guidelines to optimize these parameters for SPIM imaging of SHG nanoprobes. For instance, we focused on a specific application: tracking SHG nanoprobes seeding the blood flow in live zebrafish embryos (Figure 2). In this case, to achieve efficient detection and tracking of particles trapped in the flow that are experiencing rotational motion, it is better to mitigate the spread of SHG signals and maximize the signal mean level. For that purpose, our study demonstrates that BT nanocrystals are more adapted than KTP and that a V-polarization of the illumination and a high-NA detection should be preferred (Figure 5). We anticipate that these recommendations are not adapted for all imaging applications. Indeed, the disadvantage of KTP regarding the particle tracking application could be considered as an advantage for other applications such as those based on the orientation tracking of the particles and the design of rheology and flow shear sensors, such as reported with other types of nanoprobes. ${ }^{52}$ In this case, using a lower detection aperture will be beneficial (Figure S3) to maximize the signal sensitivity to nanoprobe orientation, and using a KTP nanocrystal with a V-polarization or BT with a $\mathrm{H}$ polarization will further maximize it (Figure 4b). Interestingly, the unique sensitivity to nanocrystal nature and rotational motion resulting from the orthogonal geometry of SHG-SPIM compared to standard imaging techniques could benefit such new applications in rheology and biological fluid dynamics.

Crystalline Nature of the Nanoprobe Used in SHGSPIM. We showed that in addition to nanoprobes size their crystalline nature is also a critical parameter for SPIM imaging. In the literature, harmonic nanocrystals are usually selected based on the mean value of the $\chi^{(2)}$ tensor coefficients. ${ }^{25}$ Indeed, the higher this value is, the larger the SHG signal becomes. We show here that the structure of this tensor also has stronger consequences in the case of orthogonal detection of SPIM than in the case of PSM (Figure 3 and Figure 4a,b). Indeed, KTP and BT nanocrystals, which have respectively high and low values of diagonal $\chi^{(2)}$ elements, exhibited different sensitivity to the illumination polarization (Figure 3 ) and rotational motion (Figure 4 and Figure S4). In addition, signal spread from KTP and BT exhibited opposite shapes and responses to light polarization or detection NA (Figure $4 \mathrm{~b}$ and Figure S3). More generally, the presence of nondiagonal elements in the $\chi^{(2)}$ tensor of the nanocrystal used results in more SHG detected in the orthogonal direction and less sensitivity to nanoprobe rotational motion and illumination polarization. Since the distribution of SHG signals strongly depends on tensor structure and differs from one nanocrystal type to the other, it should drive the choice of the SHG nanoprobe for a specific application.

\section{CONCLUSION}

Our study establishes that SHG can be efficiently used as a contrast modality for in vivo multiphoton light-sheet imaging and provides guidelines for its optimization. (i) First, unlike in point-scanning microscopy, SHG should originate from a subwavelength structure to be efficiently detected in a direction orthogonal to the illumination. In practice, we showed SHG from exogenous nanoprobes is much stronger in this geometry than endogenous SHG from biological structures. (ii) The orthogonal detection in SPIM results in more pronounced incident polarization sensitivity than in the standard collinear geometry. To enhance the average detected signal in SHG-SPIM, an illumination beam with linear polarization orthogonal to the detection axis should be preferred to a linear polarization parallel to the detection axis or even circularly polarized illumination. (iii) Moreover, the detection numerical aperture has an important effect on both the mean and the spread of detected SHG signals. In particular, it should be maximized to limit signal spread and fluctuations when tracking single nanoprobes. (iv) In addition, the crystalline nature of the nanocrystal used as SHG nanoprobe is important, especially for in vivo tracking applications in 
which nanoprobe rotational motion is involved. In this case, we provide evidence that crystalline materials exhibiting nondiagonal elements in their $\chi^{(2)}$ tensor, such as BT, are advantageous over simpler materials such as KTP. (v) Finally, we showed that SPIM imaging of SHG nanoprobes is compatible with live imaging without inducing toxicity or photodamage: by imaging live zebrafish embryos at rates up to $180 \mathrm{fps}$, it is possible to perform microangiography and resolve the blood flow profile at micrometer scales in embryonic vessels. Together, these results show that while the orthogonal geometry of light-sheet microscopy allows for fast in vivo imaging of SHG nanoprobes, it also results in a distinctive sensitivity to nanocrystal nature and particle rotational motion compared to standard imaging techniques. More generally, this work opens the way to multimodal multiphoton light-sheet microscopy combining the simultaneous detection of multiphoton excited fluorescence and coherent signals on separate channels of the same optical setup.

\section{METHODS}

Multiphoton Optical Setups. The multiphoton lightsheet (SPIM) optical setup is described in Figure 1a. Pulse trains at $80 \mathrm{MHz}$ repetition rate, at $830-960 \mathrm{~nm}$ wavelengths and with $150-180$ fs pulse durations are obtained from a Ti:sapphire laser (Chameleon Ultra II, Coherent). Beam power is adjusted using half-wave plates and polarization beam splitters. Beam size and divergence are controlled with separate telescopes. The illumination arm consists of a low NA water immersion objective (10×, $0.30 \mathrm{NA}$, Nikon), a scan lens, and a tube lens. The illumination light sheet is produced using a galvanometer mirror (Y-Scanner, GSI Lumonics) scanning the beams in the $y$-direction at $500 \mathrm{~Hz}$. The effective NA of illumination used to generate $\mathrm{a} \sim 3 \mu \mathrm{m}$ thick light-sheet is $\sim 0.1$, as previously described. ${ }^{39}$ SHG and fluorescence from the illuminated plane are collected by a high NA water immersion objective (16×, $0.80 \mathrm{NA}$, Nikon). A KG3 filter is placed immediately after the detection objective, and three spectral channels are spatially split on an electron-multiplying charged coupled device camera (EMCCD, iXon3 885, Andor) with a spectral image splitter (OptoSplit III, Cairn Research) equipped with two dichroic mirrors (FF484-FDi01 and FF560FDi01, Semrock). The objectives are mounted on translational stages for alignment. Sample is maintained in a chamber filled with a water solution and positioned from the top of the chamber with a combination of a motorized stage (MP285, Sutter Instrument) for translation in $x, y$, and $z$ directions and a rotation stage for rotation about the $y$-axis. $Z$-stack acquisition is obtained by moving the sample in the $z$ direction across the light sheet. All peripheral devices, including motorized halfwave plates, motorized delay line, shutter, galvanometer mirror, EMCCD camera, and motorized sample stage, are controlled using custom-written LabVIEW (National Instruments) software. Image acquisitions were performed with 0.67 $\mu \mathrm{m}$ per pixel, at 930 or $1060 \mathrm{~nm}$ illumination wavelength, and a mean power of 10 to $150 \mathrm{~mW}$ at the sample. For in vivo imaging, EMCCD camera acquisition was set to 2 to $10 \mathrm{~ms}$ exposure time, corresponding to 180 to $47 \mathrm{fps}$. The home-built multiphoton point-scanning microscope used to compare with SPIM was previously described in ref 53 .

Zebrafish Lines and Embryo Preparation. The following zebrafish lines were used: wild-type TL and $\mathrm{Tg}$ (gata-1:dsRed), ${ }^{54}$ provided by AMAGEN service facility at CNRS Gif-sur-Yvette (UMS 3504 CNRS/UMS 1374 INRA) and $\operatorname{Tg}(\text { fli-1:eGFP })^{50}$ crossed with $\operatorname{Tg}$ (gata-1:dsRed), kindly provided by the laboratories of Dr. Sophie Vriz (Collège de France, Paris) and Dr. Philippe Herbomel (Institut Pasteur, Paris). Embryos were raised at $28{ }^{\circ} \mathrm{C}$ in the dark and treated with 1-phenyl-2-thiourea (Sigma-Aldrich) at $10 \mathrm{hpf}$ to inhibit pigment formation. For injection or imaging, embryos were anesthetized with $0.01 \%(100 \mathrm{mg} / \mathrm{L})$ Tricaine (Sigma-Aldrich) solution and embedded in $1 \%(10 \mathrm{~g} / \mathrm{L})$ low melting point agarose (Sigma-Aldrich) as previously described. ${ }^{5}$ All experiments were performed with zebrafish embryos before independent feeding larval forms and complied with the European directive 2010/63/UE.

Preparation of KTP and BT Nanocrystals. KTP nanocrystals are negatively charged and form very stable colloidal suspensions in water, which enables their long-term storage and facilitates the sample mounting procedure. They were prepared following the protocol described in ref 33 . Briefly, two solutions of $\mathrm{Ti}(\mathrm{OBu})_{4}$ in $\mathrm{HCl}$ and $\mathrm{KH}_{2} \mathrm{PO}_{4}$ are mixed and aged for $4 \mathrm{~h}$ under ambient temperature. The solution is then neutralized up to $\mathrm{pH} 6.5$ with $\mathrm{K}_{2} \mathrm{CO}_{3}$, and the precipitate is washed with water by centrifugation. After drying, a white powder is recovered and calcined at $700{ }^{\circ} \mathrm{C}$ for $2 \mathrm{~h}$. The obtained powder is then washed with water by centrifugation and finally dispersed in pure water, leading to a colloidal suspension of KTP particles. Characterizations show that particles have an average size of about $150 \mathrm{~nm}$ and an excellent colloidal stability in water as expected from a measured zeta potential of $-40 \mathrm{mV}$ for $\mathrm{pH}$ between 6 and 8. BT nanocrystals were prepared as previously described ${ }^{35}$ and coated with polyethylene glycol to provide steric stabilization in water solution. ${ }^{35}$

Mounting and Analysis of SHG Nanoprobes in Agarose Gel. SHG nanocrystals were diluted $\times 100$ from their native solutions and sonicated using a probe sonicator using $40 \%$ power for up to $30 \mathrm{~s}$. Immediately after the sonication, sonicated particles were mixed 1:1 with $2 \%$ LMP agarose. A $20 \mu \mathrm{L}$ glass pipet was filled with the mixture, placed inside a custom-built holder, ${ }^{39}$ and mounted on the setup via a mechanical arm coupled to a motorized stage. Image analysis was performed using Imaris (Bitplane Inc.) and custom-made scripts written in Matlab (The MathWorks Inc.).

Injection and Tracking of SHG Nanoprobes in Live Zebrafish Embryos. Microangiography ${ }^{55}$ was used to deliver particles diluted as above to the zebrafish embryo circulation. To secure a consistent and harmless application of nanoprobes into the zebrafish embryo, we limited the administration of them to a single time point, since repetitive injections have been shown to be harmful to zebrafish vascular development. ${ }^{56}$ Dispensation was restricted to nanoliter volumes to prevent clogging of the small (i.e., micron-sized) injection needle that was suitable for the dimension of the zebrafish duct of Cuvier, the point of administration. Embryos were allowed $10 \mathrm{~min}$ to recover in normal embryo medium before being mounted for imaging as above. Imaging was performed in a chamber filled with embryo medium with tricaine diluted to $0.01 \%$ (100 mg/ $\mathrm{L})$ at ambient temperature. After the imaging, the embryo was recovered and raised in normal embryo medium at $28{ }^{\circ} \mathrm{C}$ to follow its development. Image sequences were analyzed using Imaris (Bitplane Inc.) and custom-made Matlab (The MathWorks Inc.) scripts to perform particle tracking and analyses of SHG signals and particle velocity.

Numerical Simulations of SHG Signals Generated by Nanocrystals and Detected Using SPIM and PSM. The 
numerical simulations of SHG signals generated by nanocrystals are based on the same theoretical framework as previously described to simulate PSM imaging of BT nanocrystals. ${ }^{43}$ It was extended to the case of KTP nanocrystals and to the orthogonal signal detection of SPIM. It relies on the following assumptions: the nanocrystals are spherical and of small size compared to the illumination wavelength; the amplitude and the phase of the electric field are uniform inside the nanocrystals and the spread inside them can be neglected. Hence, the field $\boldsymbol{E}^{\mathbf{L}}$ in the laboratory frame probed by a nanocrystal near the focus $(r, \theta, \Phi) \approx(0,0,0)$ as defined in Figure S5a is approximated as a plane wave. For a linearly polarized illumination beam, the field is given by

$$
\boldsymbol{E}^{\mathbf{L}} \propto\left(\begin{array}{l}
\cos \alpha \\
\sin \alpha \\
0
\end{array}\right) E_{0}
$$

and for a circularly polarized illumination beam, the field is given by

$$
\boldsymbol{E}^{\mathbf{L}} \propto\left(\begin{array}{l}
\frac{1}{\sqrt{2}} \\
\frac{i}{\sqrt{2}} \\
0
\end{array}\right) E_{0}
$$

where $\alpha$ is the angle of the incoming polarization compared to the $x$-axis (Figure S5a) and $E_{0}$ the amplitude of the field. For a nanocrystal whose orientation is rotated compared to the laboratory frame by the angles $\left(\phi_{\mathrm{C}}, \theta_{\mathrm{C}}, \psi_{\mathrm{C}}\right)$ as defined in Figure $\mathrm{S} 5 \mathrm{~b}$, the field in the crystal frame $E^{\mathrm{C}}$ is related to the field in the laboratory frame $E^{\mathrm{L}}$ by the Euler matrices $M\left(\phi_{\mathrm{C}}\right)$, $M\left(\theta_{\mathrm{C}}\right)$, and $M\left(\psi_{\mathrm{C}}\right)$ :

$$
\left[\begin{array}{c}
E_{X}^{\mathrm{C}} \\
E_{Y}^{\mathrm{C}} \\
E_{Z}^{\mathrm{C}}
\end{array}\right]=M\left(\psi_{\mathrm{C}}\right) M\left(\theta_{\mathrm{C}}\right) M\left(\phi_{\mathrm{C}}\right)\left[\begin{array}{c}
E_{X}^{\mathrm{L}} \\
E_{Y}^{\mathrm{L}} \\
E_{Z}^{\mathrm{L}}
\end{array}\right]
$$

where

$$
\begin{aligned}
M\left(\varphi_{\mathrm{C}}\right) & =\left[\begin{array}{lll}
\cos \phi_{\mathrm{C}} & \sin \phi_{\mathrm{C}} & 0 \\
-\sin \phi_{\mathrm{C}} & \cos \phi_{\mathrm{C}} & 0 \\
0 & 0 & 1
\end{array}\right] \\
M\left(\theta_{\mathrm{C}}\right) & =\left[\begin{array}{llll}
\cos \theta_{\mathrm{C}} & 0 & \sin \theta_{\mathrm{C}} \\
0 & 1 & 0 & \\
-\sin \theta_{\mathrm{C}} & 0 & \cos \theta_{\mathrm{C}}
\end{array}\right] \\
M\left(\psi_{\mathrm{C}}\right) & =\left[\begin{array}{llll}
\cos \psi_{\mathrm{C}} & \sin \psi_{\mathrm{C}} & 0 \\
-\sin \psi_{\mathrm{C}} & \cos \psi_{\mathrm{C}} & 0 \\
0 & 0 & 1
\end{array}\right]
\end{aligned}
$$

As introduced in ref 23, the second-order susceptibility tensor $\chi^{(2)}$ can be represented in the crystal frame as a $3 \times 6$ matrix:

$$
\begin{aligned}
\chi^{(2)} & =2\left[\begin{array}{llllll}
d_{X X X} & d_{X Y Y} & d_{X Z Z} & d_{X Y Z} & d_{X X Z} & d_{X X Y} \\
d_{Y X X} & d_{Y Y Y} & d_{Y Z Z} & d_{Y Y Z} & d_{Y X Z} & d_{Y X Y} \\
d_{Z X X} & d_{Z Y Y} & d_{Z Z Z} & d_{Z Y Z} & d_{Z X Z} & d_{Z X Y}
\end{array}\right] \\
& =2\left[\begin{array}{llllll}
d_{11} & d_{12} & d_{13} & d_{14} & d_{15} & d_{16} \\
d_{21} & d_{22} & d_{23} & d_{24} & d_{25} & d_{26} \\
d_{31} & d_{32} & d_{33} & d_{34} & d_{35} & d_{36}
\end{array}\right]
\end{aligned}
$$

For KTP nanocrystals ( $\mathrm{mm} 2$ class), the second-order polarization in the crystal frame generated by the focused field is ${ }^{23,37}$

$$
\boldsymbol{P}_{2 \omega}^{\mathbf{C}}=2\left[\begin{array}{llllll}
0 & 0 & 0 & 0 & d_{31} & 0 \\
0 & 0 & 0 & d_{32} & 0 & 0 \\
d_{31} & d_{32} & d_{33} & 0 & 0 & 0
\end{array}\right]\left[\begin{array}{l}
E_{X}^{2}\left(\boldsymbol{r}_{\boldsymbol{k}}, \omega\right) \\
E_{Y}^{2}\left(\boldsymbol{r}_{\boldsymbol{k}}, \omega\right) \\
E_{Z}^{2}\left(\boldsymbol{r}_{\boldsymbol{k}}, \omega\right) \\
2 E_{Y}\left(\boldsymbol{r}_{\boldsymbol{k}}, \omega\right) E_{Z}\left(\boldsymbol{r}_{\boldsymbol{k}}, \omega\right) \\
2 E_{X}\left(\boldsymbol{r}_{\boldsymbol{k}}, \omega\right) E_{Z}\left(\boldsymbol{r}_{\boldsymbol{k}}, \omega\right) \\
2 E_{X}\left(\boldsymbol{r}_{\boldsymbol{k}}, \omega\right) E_{Y}\left(\boldsymbol{r}_{\boldsymbol{k}}, \omega\right)
\end{array}\right]
$$

Where $\omega$ is the angular frequency of the electric field, $r_{k}$ the position within the excitation volume and $d_{31}=2.1 \mathrm{pm} / V, d_{33}$ $=15.4 \mathrm{pm} / \mathrm{V}$ and $d_{33}=15.4 \mathrm{pm} / \mathrm{V}$. In this case, the diagonal element $d_{33}$ is significantly larger than the others. In practice $|E|$ $=E_{0}$ in the crystal volume and 0 everywhere else.

For BT nanocrystals, the second order polarization in the crystal frame generated by the focused field is ${ }^{38}$

$$
\boldsymbol{P}_{\mathbf{2} \omega}^{\mathbf{C}}=2\left[\begin{array}{llllll}
0 & 0 & 0 & 0 & d_{15} & 0 \\
0 & 0 & 0 & d_{15} & 0 & 0 \\
d_{31} & d_{31} & d_{33} & 0 & 0 & 0
\end{array}\right]\left[\begin{array}{l}
E_{X}^{2}\left(\boldsymbol{r}_{\boldsymbol{k}}, \omega\right) \\
E_{Y}^{2}\left(\boldsymbol{r}_{\boldsymbol{k}}, \omega\right) \\
E_{Z}^{2}\left(\boldsymbol{r}_{\boldsymbol{k}}, \omega\right) \\
2 E_{Y}\left(\boldsymbol{r}_{\boldsymbol{k}}, \omega\right) E_{Z}\left(\boldsymbol{r}_{\boldsymbol{k}}, \omega\right) \\
2 E_{X}\left(\boldsymbol{r}_{\boldsymbol{k}}, \omega\right) E_{Z}\left(\boldsymbol{r}_{\boldsymbol{k}}, \omega\right) \\
2 E_{X}\left(\boldsymbol{r}_{\boldsymbol{k}}, \omega\right) E_{Y}\left(\boldsymbol{r}_{\boldsymbol{k}}, \omega\right)
\end{array}\right]
$$

where $d_{33}=6.7 \mathrm{pm} / \mathrm{V}, d_{31}=18 \mathrm{pm} / \mathrm{V}$, and $d_{15}=17.2 \mathrm{pm} / \mathrm{V}$. In this case, the diagonal element $d_{33}$ is significantly lower than the others.

The second-order polarization in the laboratory frame is then calculated by using the inverse of the Euler rotation matrices:

$$
\left[\begin{array}{c}
P_{X}^{\mathrm{L}}\left(\boldsymbol{r}_{\boldsymbol{k}}\right) \\
P_{Y}^{\mathrm{L}}\left(\boldsymbol{r}_{\boldsymbol{k}}\right) \\
P_{Z}^{\mathrm{L}}\left(\boldsymbol{r}_{\boldsymbol{k}}\right)
\end{array}\right]=\left(M\left(\psi_{\mathrm{C}}\right) M\left(\theta_{\mathrm{C}}\right) M\left(\phi_{\mathrm{C}}\right)\right)^{T}\left[\begin{array}{c}
P_{X}^{\mathrm{C}}\left(\boldsymbol{r}_{\boldsymbol{k}}\right) \\
P_{Y}^{\mathrm{C}}\left(\boldsymbol{r}_{\boldsymbol{k}}\right) \\
P_{Z}^{\mathrm{C}}\left(\boldsymbol{r}_{\boldsymbol{k}}\right)
\end{array}\right]
$$

The harmonic field originating from all the positions $r_{k}$ in the nanocrystal and propagating to a position $r=(r, \theta, \phi)$ is given by $^{37,57}$

$$
\boldsymbol{E}_{\mathbf{S H G}}(\boldsymbol{r})=-\frac{(2 \omega)^{2}}{c^{2}} \iiint \mathrm{d} V_{k} \boldsymbol{G}_{\mathbf{0}}\left(\boldsymbol{r}, \boldsymbol{r}_{\boldsymbol{k}}\right)\left[\begin{array}{c}
P_{X}^{\mathrm{L}}\left(\boldsymbol{r}_{\boldsymbol{k}}\right) \\
P_{Y}^{\mathrm{L}}\left(\boldsymbol{r}_{\boldsymbol{k}}\right) \\
P_{Z}^{\mathrm{L}}\left(\boldsymbol{r}_{\boldsymbol{k}}\right)
\end{array}\right]
$$


where $V_{k}$ is nanocrystal volume and $G_{0}$ the far-field Green's function: ${ }^{57}$

$$
\begin{aligned}
& \boldsymbol{G}_{\mathbf{0}}\left(\boldsymbol{r}, \boldsymbol{r}_{\boldsymbol{k}}\right)=\frac{\exp \left(i k_{2 \omega} r\right)}{4 \pi r} \exp \left(-i k_{2 \omega} \frac{\boldsymbol{r} \cdot \boldsymbol{r}_{\boldsymbol{k}}}{r}\right) \\
& \times\left[\begin{array}{lll}
1-\cos ^{2} \phi \sin ^{2} \theta & -\sin \phi \cos \phi \sin ^{2} \theta & -\cos \phi \sin \theta \cos \theta \\
-\sin \phi \cos \phi \sin ^{2} \theta & 1-\sin ^{2} \phi \sin ^{2} \theta & -\sin \phi \sin \theta \cos \theta \\
-\cos \phi \sin \theta \cos \theta & -\sin \phi \sin \theta \cos \theta & \sin ^{2} \theta
\end{array}\right]
\end{aligned}
$$

Finally, the SHG power $P_{S H G}$ radiated by a nanocrystal and collected over the cone angle $\Omega$ (Figure S5c,d) in PSM is given by

$$
P_{\mathrm{SHG}}=\int_{0}^{2 \pi} \int_{0}^{\Omega}\left|\boldsymbol{E}_{\mathrm{SHG}}(\boldsymbol{r})\right|^{2} \sin (\theta) \mathrm{d} \theta \mathrm{d} \phi
$$

and in SPIM, it is given by

$$
P_{\mathrm{SHG}}=\int_{0}^{\Omega} \int_{0}^{2 \pi}\left|\boldsymbol{E}_{\mathrm{SHG}}(\boldsymbol{r})\right|^{2} \sin (\theta) \mathrm{d} \theta \mathrm{d} \phi
$$

For all numerical simulations, we used an excitation wavelength $\lambda=930 \mathrm{~nm}$, a particle diameter of one voxel set to $36.9 \mathrm{~nm}$, and random crystal orientation angles $\phi_{\mathrm{c}}$ and $\theta_{\mathrm{c}}$ (uniformly distributed on a sphere). The detection numerical aperture was $0.8,1.0$, or 1.33 , corresponding to angle $\Omega=\operatorname{asin} \frac{\mathrm{NA}}{n_{\text {Water }}}=37^{\circ}, 49^{\circ}$, or $90^{\circ}$, respectively. The excitation polarization was linear or circular, and for each simulation, we computed the total collected SHG power for each excitation polarization for $N$ randomly orientated nanocrystals.

Experimental Estimation of SHG Signal Levels Using Single-Particle Tracking. To compare the histograms of signal levels between experiments and simulations (Figure 5), we took into account the variability of nanocrystal sizes in the experiments. To remove the effect of nanocrystal size variability, we tracked individual nanoprobes. We selected only the nanoprobes that were detected at least 30 times during their flow inside the blood vessel. Then, we normalized the signal to the maximal intensity found along each individual trajectory. The signals (between 0 and 1 ) from different nanoprobes were then used to plot the histograms of Figure 5c,d (2693 and 767 values for KTP and BT nanocrystals, respectively). Such a histogram should be comparable to simulated histograms assuming along a track each nanoprobe reaches an orientation corresponding to a maximum of detected signal. To generate simulated histograms (Figure $5 e, f)$, we estimated the distribution of signal detected from 10000 nanocrystals with random (uniformly distributed) orientations in the SPIM geometry with a 0.8 detection NA. The signals were normalized to the maximum. To take into account the effect of the Gaussian light-sheet profile in the axial direction (Figure 5g,h), we multiplied the signal with a Gaussian before normalization. We adjusted the range of explored axial positions to obtain the best match between the experimental and the simulated histograms in the case of BT nanocrystals (Figure $5 \mathrm{~d}$ and $\mathrm{f}$, respectively). Its value corresponds to nanocrystals exploring $\pm 7 \mu \mathrm{m}$ around the center of a light-sheet of $4 \mu \mathrm{m}$ full width half-maximum, which is consistent with both the observed lateral movements of the nanocrystals along a track and the blood vessel diameters.

\section{ASSOCIATED CONTENT}

\section{Supporting Information}

The Supporting Information is available free of charge at https://pubs.acs.org/doi/10.1021/acsphotonics.9b01749.

Supporting movie 1 (AVI)

Supporting movie 2 (AVI)

Supporting movie 3 (AVI)

Supporting movie 4 (AVI)

Supporting figures and supporting results (PDF)

\section{AUTHOR INFORMATION}

\section{Corresponding Authors}

Emmanuel Beaurepaire - Laboratory for Optics and Biosciences, Ecole Polytechnique, CNRS, INSERM, Université Paris-Saclay, 91128 Palaiseau Cedex, France; 이이.org/ 0000-0002-2082-8214; Email: emmanuel.beaurepaire@ polytechnique.edu

Willy Supatto - Laboratory for Optics and Biosciences, Ecole Polytechnique, CNRS, INSERM, Universite Paris-Saclay, 91128 Palaiseau Cedex, France; 이이이.org/0000-0002-4562-9166; Email: willy.supatto@polytechnique.edu

\section{Authors}

Guy Malkinson - Laboratory for Optics and Biosciences, Ecole Polytechnique, CNRS, INSERM, Université Paris-Saclay, 91128 Palaiseau Cedex, France; (1) orcid.org/0000-0001-7339-3659

Pierre Mahou - Laboratory for Optics and Biosciences, Ecole Polytechnique, CNRS, INSERM, Universite Paris-Saclay, 91128 Palaiseau Cedex, France; ○ orcid.org/0000-0003-4452-1011

Élodie Chaudan - Laboratory of Condensed Matter Physics, Ecole Polytechnique, CNRS, Université Paris-Saclay, 91128 Palaiseau Cedex, France

Thierry Gacoin - Laboratory of Condensed Matter Physics, Ecole Polytechnique, CNRS, Universite Paris-Saclay, 91128 Palaiseau Cedex, France; 이이.org/0000-0001-6774-3181

Ali Y. Sonay - Department of Biosystems Science and Engineering (D-BSSE), Eidgenössische Technische Hochschule (ETH) Zurich, 4058 Basel, Switzerland; 다이.org/00000002-2194-9958

Periklis Pantazis - Department of Biosystems Science and Engineering (D-BSSE), Eidgenössische Technische Hochschule (ETH) Zurich, 4058 Basel, Switzerland; Department of Bioengineering, Imperial College London, London SW7 2AZ, U.K.; ○ orcid.org/0000-0002-8367-9332

Complete contact information is available at:

https://pubs.acs.org/10.1021/acsphotonics.9b01749

\section{Author Contributions}

${ }^{\perp}$ G. Malkinson and P. Mahou contributed equally to this work. 


\section{Author Contributions}

G.M., P.M., E.B., and W.S. conceived the project. G.M. and P.M. performed the experiments and simulations. E.C. and T.G. produced the KTP nanocrystal suspension. A.Y.S. and P.P. produced the BT nanocrystal suspension. G.M., P.M., and W.S. analyzed and processed the data. W.S. designed the figures. All authors contributed to the manuscript text and have given approval to the final version of the manuscript.

\section{Notes}

The authors declare no competing financial interest.

\section{ACKNOWLEDGMENTS}

This work was supported by Agence Nationale de la Recherche (ANR-11-EQPX-0029 Morphoscope2, ANR-2010-JCJC-151001, ANR-10-INBS-04 France BioImaging), by the European Union Seventh Framework Programme (Marie Curie International Reintegration grant no. 268379), by Ecole Polytechnique, and by Région Ile-de-France. Work in the lab of P.P. was supported by a Royal Society Wolfson Research Merit Award. We thank Lamiae Abdeladim, Marie-Claire Schanne-Klein, Joséphine Morizet, Chiara Stingari, and other LOB members for scientific discussions. We thank Emilie Menant for assistance with zebrafish husbandary. We thank the AMAGEN facility at CNRS Gif-sur-Yvette (UMS 3504 CNRS/UMS 1374 INRA), Dr. Sophie Vriz (Collège de France, Paris), and Dr. Philippe Herbomel (Institut Pasteur, Paris) for providing zebrafish embryos.

\section{REFERENCES}

(1) Huisken, J.; Swoger, J.; Del Bene, F.; Wittbrodt, J.; Stelzer, E. H. Optical sectioning deep inside live embryos by selective plane illumination microscopy. Science 2004, 305 (5686), 1007-9.

(2) Keller, P. J.; Schmidt, A. D.; Wittbrodt, J.; Stelzer, E. H. Reconstruction of zebrafish early embryonic development by scanned light sheet microscopy. Science 2008, 322 (5904), 1065-9.

(3) Mertz, J. Optical sectioning microscopy with planar or structured illumination. Nat. Methods 2011, 8, 811.

(4) Pantazis, P.; Supatto, W. Advances in whole-embryo imaging: a quantitative transition is underway. Nat. Rev. Mol. Cell Biol. 2014, 15 (5), 327-39.

(5) Truong, T. V.; Supatto, W.; Koos, D. S.; Choi, J. M.; Fraser, S. E. Deep and fast live imaging with two-photon scanned light-sheet microscopy. Nat. Methods 2011, 8 (9), 757-760.

(6) Supatto, W.; Truong, T. V.; Débarre, D.; Beaurepaire, E. Advances in multiphoton microscopy for imaging embryos. Curr. Opin. Genet. Dev. 2011, 21 (5), 538-48.

(7) Pavone, F. S.; Campagnola, P. J. Second Harmonic Generation Imaging; CRC Press, 2016; p 476.

(8) Chen, X.; Nadiarynkh, O.; Plotnikov, S.; Campagnola, P. J. Second harmonic generation microscopy for quantitative analysis of collagen fibrillar structure. Nat. Protoc. 2012, 7, 654.

(9) Boulesteix, T.; Beaurepaire, E.; Sauviat, M. P.; Schanne-Klein, M. C. Second-harmonic microscopy of unstained living cardiac myocytes: measurements of sarcomere length with 20-nm accuracy. Opt. Lett. 2004, 29 (17), 2031-2033.

(10) Dombeck, D. A.; Kasischke, K. A.; Vishwasrao, H. D.; Ingelsson, M.; Hyman, B. T.; Webb, W. W. Uniform polarity microtubule assemblies imaged in native brain tissue by secondharmonic generation microscopy. Proc. Natl. Acad. Sci. U. S. A. 2003, 100 (12), 7081-7086.

(11) Moreaux, L.; Sandre, O.; Charpak, S.; Blanchard-Desce, M.; Mertz, J. Coherent scattering in multi-harmonic light microscopy. Biophys. J. 2001, 80 (3), 1568-1574.

(12) Zipfel, W. R.; Williams, R. M.; Webb, W. W. Nonlinear magic: multiphoton microscopy in the biosciences. Nat. Biotechnol. 2003, 21, 1369.
(13) Rouede, D.; Bellanger, J. J.; Schaub, E.; Recher, G.; Tiaho, F. Theoretical and Experimental SHG Angular Intensity Patterns from Healthy and Proteolysed Muscles. Biophys. J. 2013, 104 (9), 19591968.

(14) Gusachenko, I.; Schanne-Klein, M. C. Numerical simulation of polarization-resolved second-harmonic microscopy in birefringent media. Phys. Rev. A: At., Mol., Opt. Phys. 2013, 88 (5), 15.

(15) Houle, M. A.; Couture, C. A.; Bancelin, S.; Van der Kolk, J.; Auger, E.; Brown, C.; Popov, K.; Ramunno, L.; Legare, F. Analysis of forward and backward Second Harmonic Generation images to probe the nanoscale structure of collagen within bone and cartilage. J. Biophotonics 2015, 8 (11-12), 993-1001.

(16) Dempsey, W. P.; Fraser, S. E.; Pantazis, P. SHG nanoprobes: Advancing harmonic imaging in biology. BioEssays 2012, 34 (5), $351-360$.

(17) Staedler, D.; Magouroux, T.; Hadji, R.; Joulaud, C. c.; Extermann, J. r. m.; Schwung, S.; Passemard, S. n.; Kasparian, C.; Clarke, G.; Gerrmann, M.; Dantec, R. L.; Mugnier, Y.; Rytz, D.; Ciepielewski, D.; Galez, C.; Gerber-Lemaire, S.; Juillerat-Jeanneret, L.; Bonacina, L.; Wolf, J.-P. Harmonic Nanocrystals for Biolabeling: A Survey of Optical Properties and Biocompatibility. ACS Nano 2012, 6 (3), 2542-2549.

(18) Magouroux, T.; Extermann, J.; Hoffmann, P.; Mugnier, Y.; Le Dantec, R.; Jaconi, M. E.; Kasparian, C.; Ciepielewski, D.; Bonacina, L.; Wolf, J.-P. High-Speed Tracking of Murine Cardiac Stem Cells by Harmonic Nanodoublers. Small 2012, 8 (17), 2752-2756.

(19) Debarre, D.; Olivier, N.; Beaurepaire, E. Signal epidetection in third-harmonic generation microscopy of turbid media. Opt. Express 2007, 15 (14), 8913-24.

(20) Cheng, J. X.; Jia, Y. K.; Zheng, G.; Xie, X. S. Laser-scanning coherent anti-Stokes Raman scattering microscopy and applications to cell biology. Biophys. J. 2002, 83 (1), 502-9.

(21) Le Dantec, R.; Mugnier, Y.; Djanta, G.; Bonacina, L.; Extermann, J.; Badie, L.; Joulaud, C.; Gerrmann, M.; Rytz, D.; Wolf, J. P.; Galez, C. Ensemble and Individual Characterization of the Nonlinear Optical Properties of $\mathrm{ZnO}$ and BaTiO3 Nanocrystals. J. Phys. Chem. C 2011, 115 (31), 15140-15146.

(22) Joulaud, C.; Mugnier, Y.; Djanta, G.; Dubled, M.; Marty, J.-C.; Galez, C.; Wolf, J.-P.; Bonacina, L.; Le Dantec, R. Characterization of the nonlinear optical properties of nanocrystals by Hyper Rayleigh Scattering. J. Nanobiotechnol. 2013, 11 (1), S8.

(23) Boyd, R. W. Chapter 1 - The Nonlinear Optical Susceptibility. In Nonlinear Optics, second ed.; Academic Press: San Diego, 2003; pp 1-65, DOI: 10.1016/B978-012121682-5/50002-X.

(24) Brasselet, S.; Zyss, J. Nano-Crystals for Quadratic Nonlinear Imaging: Characterization and Applications. In Nanotechnology and Nanomaterials "Nanocrystals"; Masuda, Y., Ed.; Sciyo, 2010.

(25) Rogov, A.; Mugnier, Y.; Bonacina, L. Harmonic nanoparticles: noncentrosymmetric metal oxides for nonlinear optics. J. Opt. 2015, 17 (3), 033001.

(26) Nakayama, Y.; Pauzauskie, P. J.; Radenovic, A.; Onorato, R. M.; Saykally, R. J.; Liphardt, J.; Yang, P. Tunable nanowire nonlinear optical probe. Nature 2007, 447 (7148), 1098-1101.

(27) Extermann, J.; Bonacina, L.; Cuøa, E.; Kasparian, C.; Mugnier, Y.; Feurer, T.; Wolf, J.-P. Nanodoublers as deep imaging markers for multi-photon microscopy. Opt. Express 2009, 17 (17), 15342-15349.

(28) Pu, Y.; Hsieh, C.-L.; Grange, R.; Yang, X.; Papadopoulos, I.; Choi, J.-W.; Psaltis, D. Second harmonic nanoparticles in imaging applications. Proc. SPIE 2011, 8095, 80950E-80950E-9.

(29) Ahmed, F. Laser damage threshold of KTiOPO4. Appl. Opt. 1989, 28, 119-122.

(30) Hildenbrand, a.; Wagner, F. R.; Akhouayri, H.; Natoli, J.-Y.; Commandré, M.; Théodore, F.; Albrecht, H. Laser-induced damage investigation at $1064 \mathrm{~nm}$ in KTiOPO4 crystals and its analogy with RbTiOPO4. Appl. Opt. 2009, 48, 4263-4269.

(31) Le Xuan, L.; Zhou, C.; Slablab, A.; Chauvat, D.; Tard, C.; Perruchas, S.; Gacoin, T.; Villeval, P.; Roch, J.-F. Photostable SecondHarmonic Generation from a Single KTiOPO4 Nanocrystal for Nonlinear Microscopy. Small 2008, 4 (9), 1332-1336. 
(32) Pantazis, P.; Maloney, J.; Wu, D.; Fraser, S. E. Second harmonic generating (SHG) nanoprobes for in vivo imaging. Proc. Natl. Acad. Sci. U. S. A. 2010, 107 (33), 14535-40.

(33) Mayer, L.; Slablab, A.; Dantelle, G.; Jacques, V.; LepagnolBestel, A.-M.; Perruchas, S.; Spinicelli, P.; Thomas, A.; Chauvat, D.; Simonneau, M.; Gacoin, T.; Roch, J.-F. Single KTP nanocrystals as second-harmonic generation biolabels in cortical neurons. Nanoscale 2013, 5 (18), 8466-8471.

(34) Mahou, P.; Malkinson, G.; Chaudan, É.; Gacoin, T.; Beaurepaire, E.; Supatto, W. Metrology of Multiphoton Microscopes Using Second Harmonic Generation Nanoprobes. Small 2017, 13, (42), 1701442.

(35) Sugiyama, N.; Sonay, A. Y.; Tussiwand, R.; Cohen, B. E.; Pantazis, P. Effective Labeling of Primary Somatic Stem Cells with BaTiO3 Nanocrystals for Second Harmonic Generation Imaging. Small 2018, 14 (8), 1703386.

(36) Culic-Viskota, J.; Dempsey, W. P.; Fraser, S. E.; Pantazis, P. Surface functionalization of barium titanate SHG nanoprobes for in vivo imaging in zebrafish. Nat. Protoc. 2012, 7 (9), 1618-33.

(37) Pack, M. V.; Armstrong, D. J.; Smith, A. V. Measurement of the Chi(2) tensors of KTiOPO4, KTiOAsO4, RbTiOPO4, and RbTiOAsO4 crystals. Appl. Opt. 2004, 43 (16), 3319-3323.

(38) Bohren, C. F.; Huffman, D. R. Absorption and Scattering of Light by Small Particles; Wiley, 1998; DOI: 10.1002/9783527618156.

(39) Mahou, P.; Vermot, J.; Beaurepaire, E.; Supatto, W. Multicolor two-photon light-sheet microscopy. Nat. Methods 2014, 11 (6), 6001.

(40) Kim, E.; Steinbrück, A.; Buscaglia, M. T.; Buscaglia, V.; Pertsch, T.; Grange, R. Second-Harmonic Generation of Single BaTiO3 Nanoparticles down to $22 \mathrm{~nm}$ Diameter. ACS Nano 2013, 7 (6), 5343-5349.

(41) Le Floc'h, V.; Brasselet, S.; Roch, J.-F.; Zyss, J. Monitoring of Orientation in Molecular Ensembles by Polarization Sensitive Nonlinear Microscopy. J. Phys. Chem. B 2003, 107, 12403-12410.

(42) Brasselet, S.; FloC'H, V. L.; Treussart, F.; Roch, J. F.; Zyss, J.; Botzung-Appert, E.; Ibanez, A. In situ diagnostics of the crystalline nature of single organic nanocrystals by nonlinear microscopy. Phys. Rev. Lett. 2004, 92, 207401-1.

(43) Hsieh, C.-L.; Pu, Y.; Grange, R.; Psaltis, D. Second harmonic generation from nanocrystals under linearly and circularly polarized excitations. Opt. Express 2010, 18 (11), 11917-11932.

(44) Dempsey, W. P.; Hodas, N. O.; Ponti, A.; Pantazis, P. Determination of the source of SHG verniers in zebrafish skeletal muscle. Sci. Rep. 2015, 5, 18119.

(45) Anton, H.; Harlepp, S.; Ramspacher, C.; Wu, D.; Monduc, F.; Bhat, S.; Liebling, M.; Paoletti, C.; Charvin, G.; Freund, J. B.; Vermot, J. Pulse propagation by a capacitive mechanism drives embryonic blood flow. Development 2013, 140 (21), 4426-34.

(46) Boselli, F.; Freund, J. B.; Vermot, J. Blood flow mechanics in cardiovascular development. Cell. Mol. Life Sci. 2015, 72 (13), 254559.

(47) Sugden, W. W.; Meissner, R.; Aegerter-Wilmsen, T.; Tsaryk, R.; Leonard, E. V.; Bussmann, J.; Hamm, M. J.; Herzog, W.; Jin, Y.; Jakobsson, L.; Denz, C.; Siekmann, A. F. Endoglin controls blood vessel diameter through endothelial cell shape changes in response to haemodynamic cues. Nat. Cell Biol. 2017, 19 (6), 653-665.

(48) Supatto, W.; Débarre, D.; Farge, E.; Beaurepaire, E. Femtosecond pulse-induced microprocessing of live Drosophila embryos. Medical Laser Application 2005, 20 (3), 207-216.

(49) Baker, K.; Warren, K. S.; Yellen, G.; Fishman, M. C. Defective "pacemaker" current $\left(I_{\mathrm{h}}\right)$ in a zebrafish mutant with a slow heart rate. Proc. Natl. Acad. Sci. U. S. A. 1997, 94 (9), 4554-4559.

(50) Lawson, N. D.; Weinstein, B. M. In vivo imaging of embryonic vascular development using transgenic zebrafish. Dev. Biol. 2002, 248 (2), 307-18.

(51) Strupler, M.; Pena, A. M.; Hernest, M.; Tharaux, P. L.; Martin, J. L.; Beaurepaire, E.; Schanne-Klein, M. C. Second harmonic imaging and scoring of collagen in fibrotic tissues. Opt. Express 2007, 15 (7), 4054-65.
(52) Kim, J.; Michelin, S.; Hilbers, M.; Martinelli, L.; Chaudan, E.; Amselem, G.; Fradet, E.; Boilot, J.-P.; Brouwer, A. M.; Baroud, C. N.; Peretti, J.; Gacoin, T. Monitoring the orientation of rare-earth-doped nanorods for flow shear tomography. Nat. Nanotechnol. 2017, 12, 914.

(53) Zimmerley, M.; Mahou, P.; Débarre, D.; Schanne-Klein, M.-C.; Beaurepaire, E. Probing Ordered Lipid Assemblies with Polarized Third-Harmonic-Generation Microscopy. Phys. Rev. X 2013, 3 (1), 011002 .

(54) Traver, D.; Paw, B. H.; Poss, K. D.; Penberthy, W. T.; Lin, S.; Zon, L. I. Transplantation and in vivo imaging of multilineage engraftment in zebrafish bloodless mutants. Nat. Immunol. 2003, 4 (12), 1238-46.

(55) Kamei, M.; Isogai, S.; Weinstein, B. M. Imaging blood vessels in the zebrafish. Methods Cell Biol. 2004, 76, 51-74.

(56) Brown, H. K.; Schiavone, K.; Tazzyman, S.; Heymann, D.; Chico, T. J. Zebrafish xenograft models of cancer and metastasis for drug discovery. Expert Opin. Drug Discovery 2017, 12 (4), 379-389.

(57) Novotny, L.; Hecht, B. Principles of Nano-Optics; Cambridge University Press: Cambridge, 2006, DOI: 10.1017/ CBO9780511813535. 


\title{
Fast in vivo imaging of SHG nanoprobes with
}

\section{multiphoton light-sheet microscopy}

\author{
Guy Malkinsonł: Pierre Mahouł, Élodie Chaudan, Thierry Gacoin, Ali Y. Sonay, \\ Periklis Pantazis, Emmanuel Beaurepaire, and Willy Supatto \\ \$ These authors contributed equally to this work.
}

\section{SUPPORTING INFORMATION}

11 pages

5 Supporting Figures (Figure S1-S5)

4 Supporting Movies (Movie 1-4)

2 Supporting Results 
a
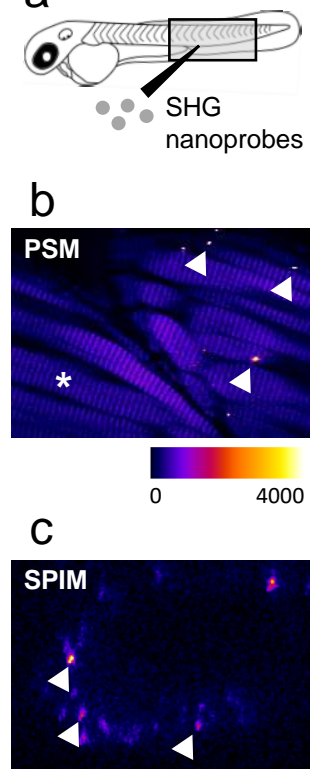

ill.
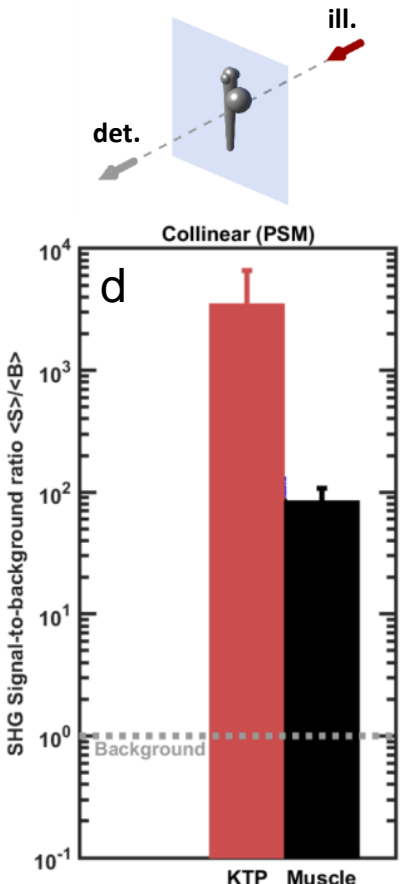

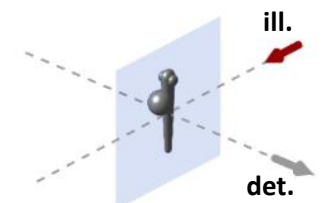

ill.

det.

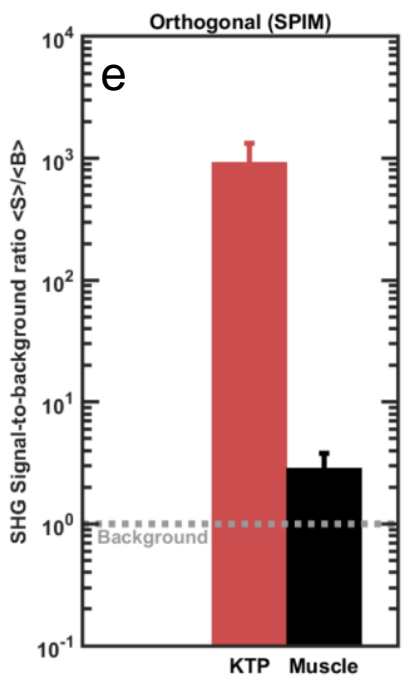

FIGURE S1

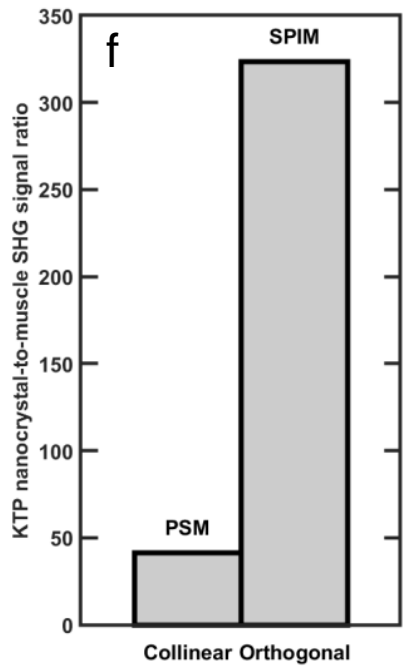

Figure S1. Comparison of SHG detection efficiency using orthogonal (SPIM) or collinear (PSM) geometry to image SHG nanocrystals or endogenous sources of SHG. (a) KTP nanocrystals were injected to the zebrafish embryo tail muscle. (b-c) Single z-stacks of a zebrafish embryo were taken in collinear (b) and orthogonal (c) geometry. SHG signals generated by the skeletal muscle, seen as vertical striations, were detected only using PSM (b), whereas signals from SHG nanoprobes, seen as small localized points (white arrows) were detected in both cases (b-c). (d-f) Quantification of the data shown in (b-c), respectively. For each type of geometry, the mean intensity values through the 3D z-stack are given for the KTP nanocrystals (red) and for muscle region (black). Note that in the orthogonal geometry (c and e), The SHG signals from muscle were hardly visible and only slightly above the background level (gray dotted line). The KTP nanocrystal-to-muscle SHG signal ratio (f) obtained from these measurements showed a pronounced difference between collinear and orthogonal geometry: when using PSM imaging as a reference, SHG signals from KTP nanocrystals were an order of magnitude more efficiently detected than signals from muscles using SPIM. ill., illumination; det. detection. 

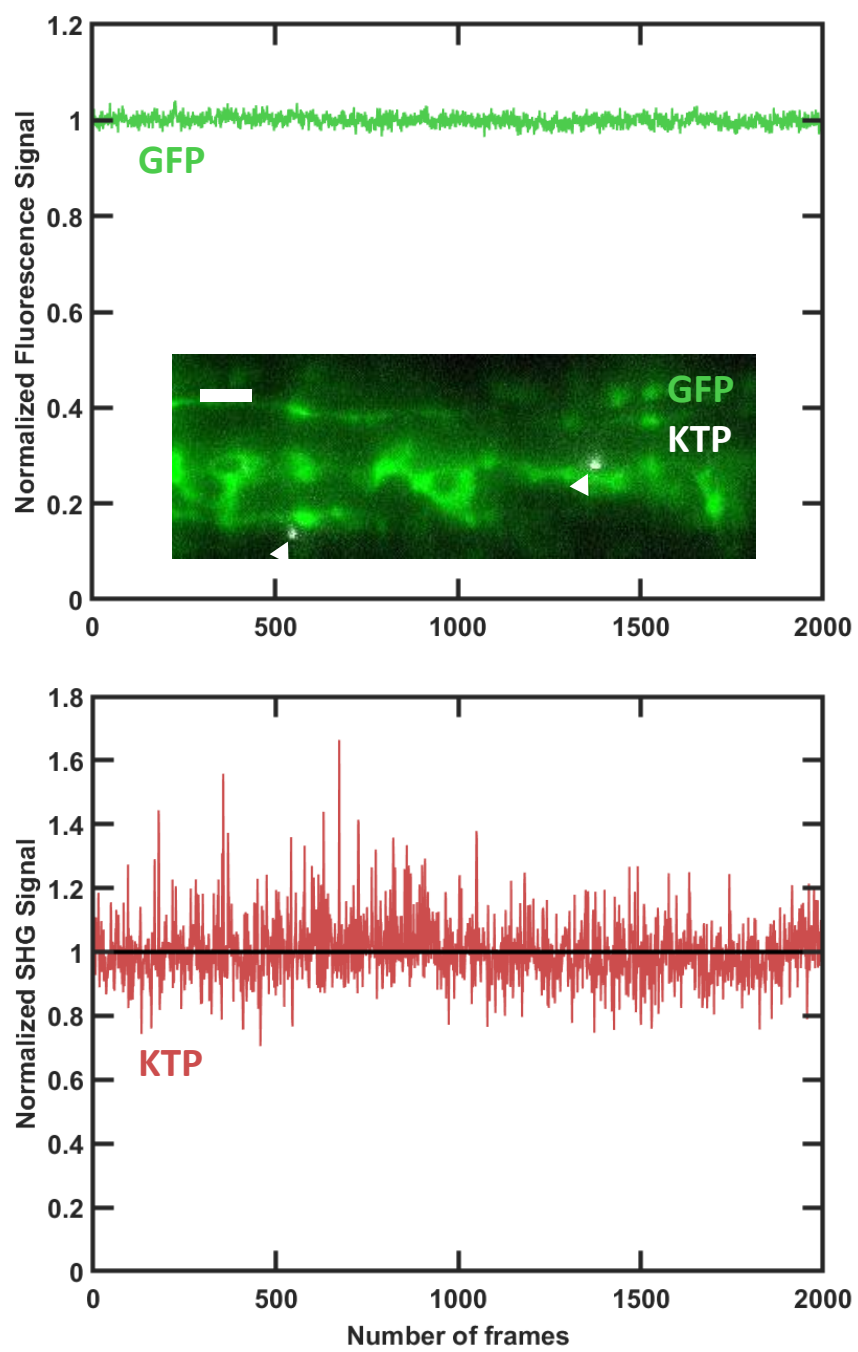

FIGURE S2

Figure S2. Photostability of fluorescence and SHG signals during multimodal multiphoton in vivo imaging. Graphs of fluorescence signals from GFP (green, top) and SHG signals from KTP (red, bottom) normalized to the mean depending the number of acquired frames during multimodal multiphoton imaging (inset, fluorescence signal in green and KTP nanocrystals in gray indicated with white arrowheads, scale bar $20 \mu \mathrm{m}$ ). Image acquired at $75 \mathrm{fps}, 10 \mathrm{~ms}$ exposure time, $930 \mathrm{~nm}$ illumination wavelength and $150 \mathrm{~mW}$ mean power. 

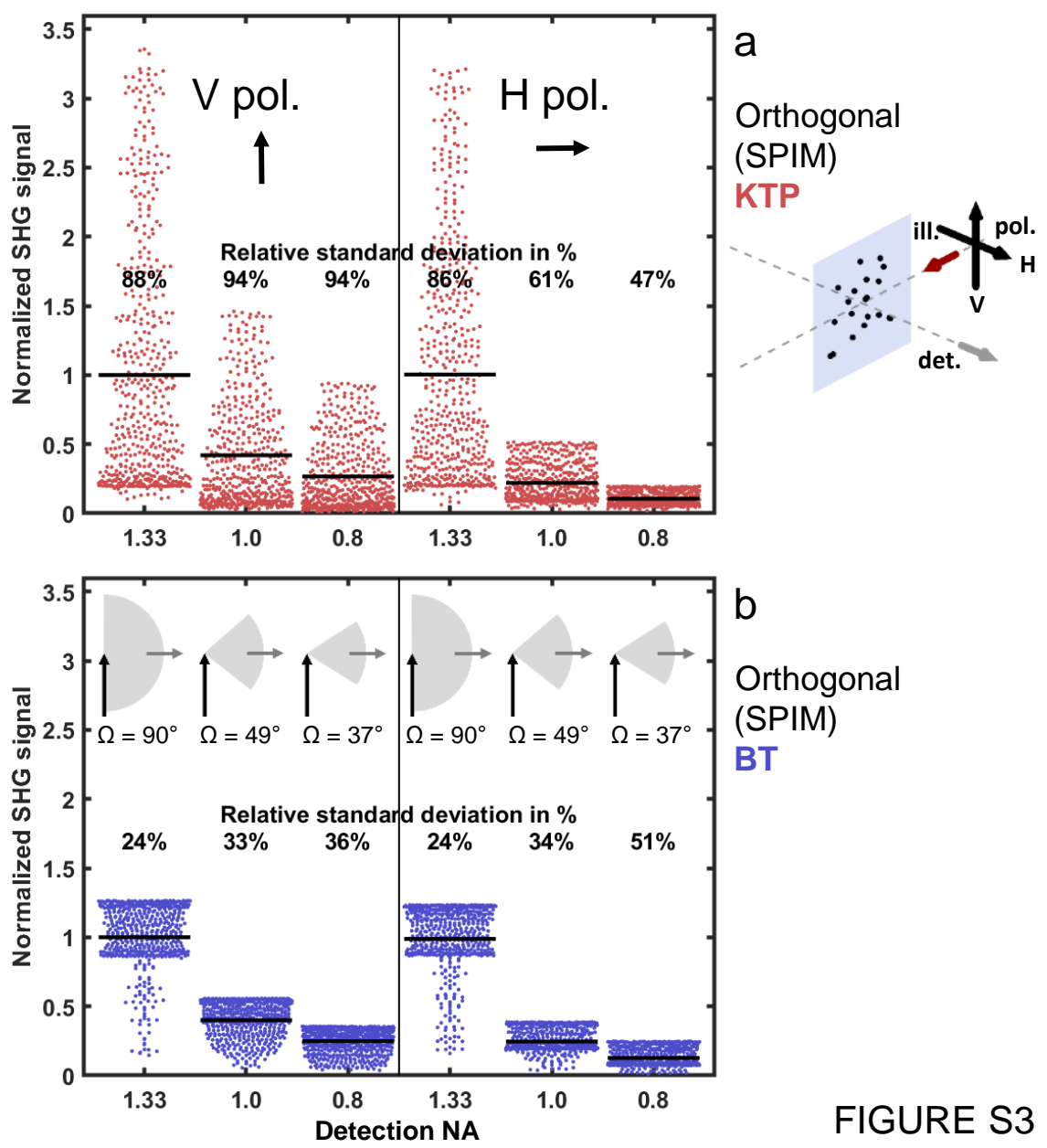

Figure S3. Simulation of SHG signals detected from individual KTP and BT nanocrystals depending on detection numerical aperture (NA). Simulation of SHG signal distributions from 500 KTP (a, red) and BT (b, blue) nanocrystals using linear vertical (V, left) or linear horizontal (H, right) polarization of the illumination light in orthogonal geometry of SPIM. Distributions are plotted depending on the detection NA $\left(0.8,1.0\right.$ or 1.33 values, corresponding to 90,49 and $37^{\circ}$ semi-cone angle $\Omega$ of detection). Random distribution of nanocrystal orientations results in spreading of signal levels around the mean (black horizontal lines). Signal for each polarization is normalized to the signal mean in the 1.33 detection NA case. Note: $\mathrm{NA}=1.33$ corresponds to the total radiated SHG signal and can be directly compared to PSM. The 24\% relative standard deviation in the case of BT is consistent with previous work obtained in PSM $^{39}$. Signal mean and relative standard deviation are indicated for 10,000 nanocrystals simulated with random orientations. 
ill pol. Orthogonal

FIGURE S4

$\because \because$

$$
\text { det. }
$$
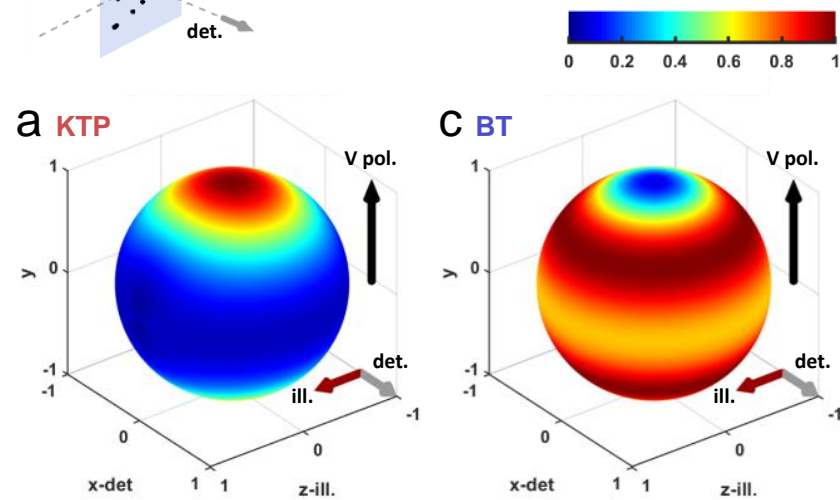

C BT
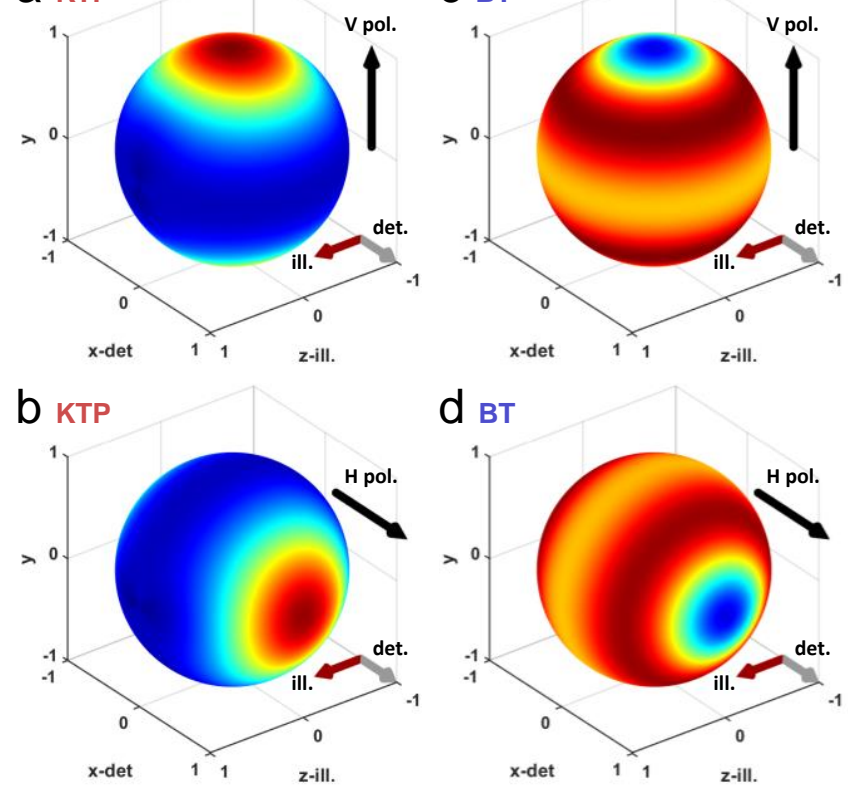

$\mathrm{d}_{\mathrm{BT}}$

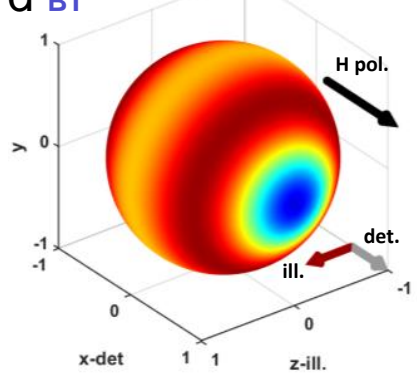

Total SHG

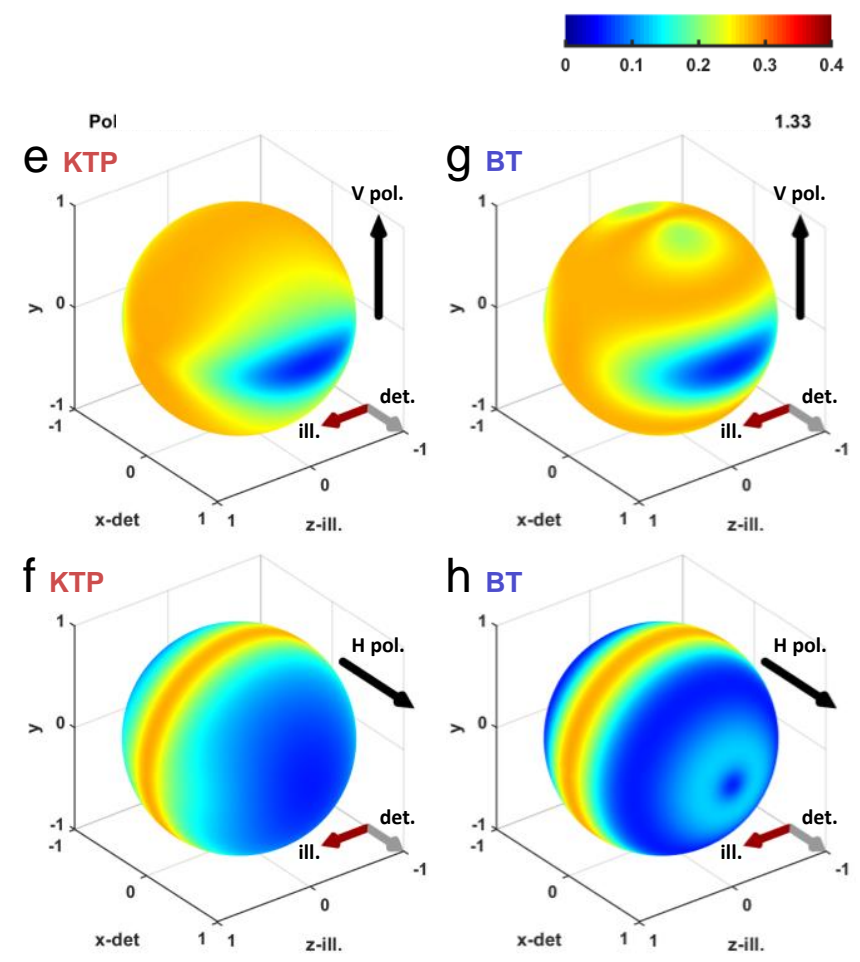

Signal collection efficiency 
Figure S4. Simulation of total SHG generated and signal collection efficiency depending on KTP and BT nanocrystal orientation and linear polarization of the illumination light in lightsheet microscopy. (a-d) Simulation of signal intensity distribution for all possible nanocrystal orientations for KTP (a-b) and BT (c-d) nanocrystals using vertical (a and c) or horizontal (b and d) linear polarization in SPIM with a 1.33 detection NA. Signals were calculated, displayed and normalized the same way as Figure 4d-g, except for the detection NA value. The plot corresponds to the distribution of the total SHG signal radiated by the nanoprobes. While KTP nanocrystals behave as a single dipole, the BT nanocrystal radiates a constant signal almost independently on its 3D orientation. (e-h) signal collection efficiency is estimated by computing the ratio of simulations using a 0.8 (Figure 4d-g) and a 1.33 (a-d) detection NA before signal normalization. These graphs show that using a H-polarization result in a poor signal collection efficiency when using SPIM for almost all nanocrystal orientations. Red and gray arrows corresponds to the SPIM illumination and detection axes, respectively. Represented signal is a spline interpolation of signals from 4,000 nanocrystals simulated with random orientations. ill., illumination; det. detection; V, vertical; H, horizontal. 

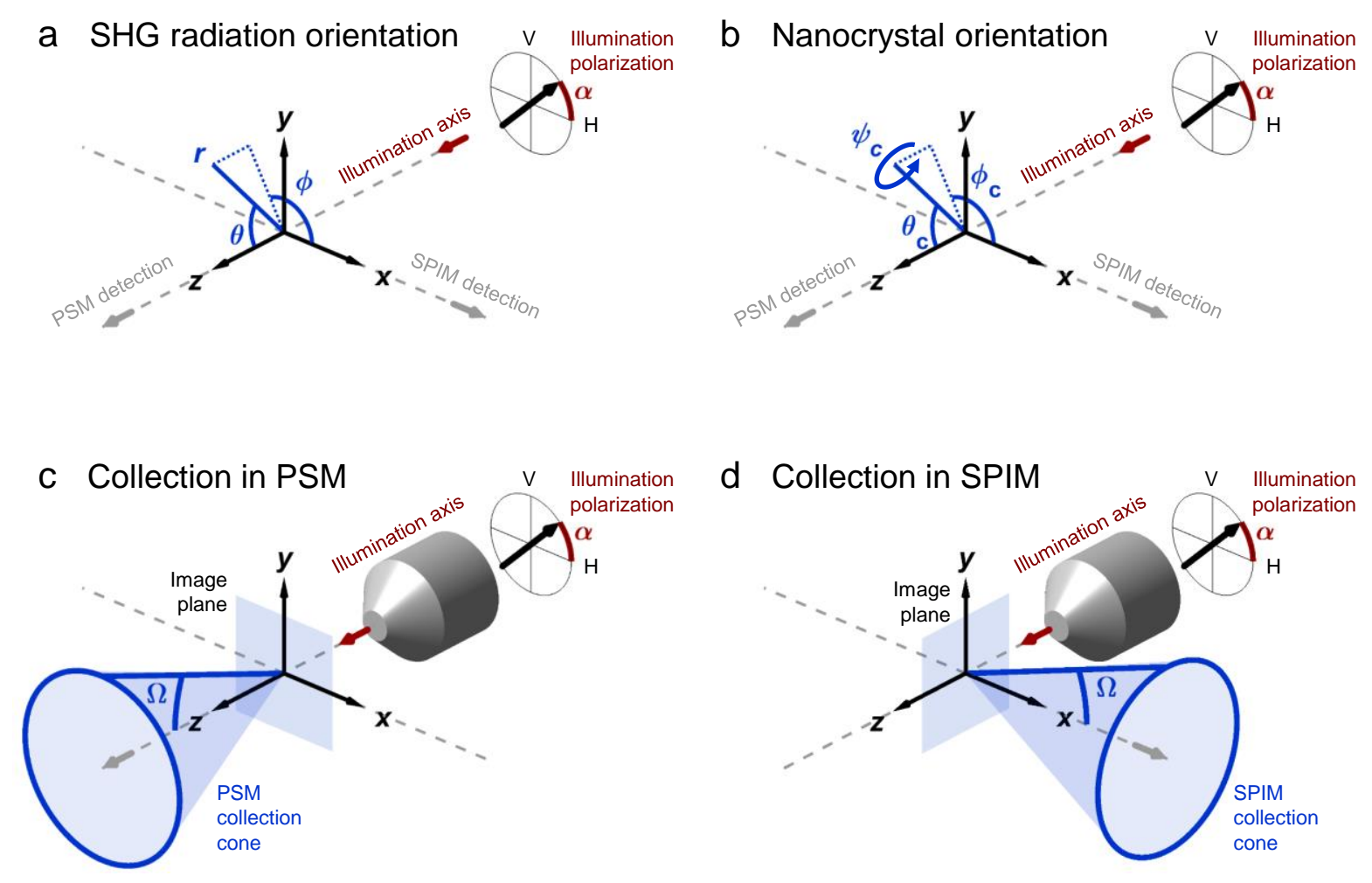

FIGURE S5

Figure S5. Spatial coordinate systems used in simulations. (a) $(x, y, z)$ and $(r, \theta, \varphi)$ are the Cartesian and spherical coordinate system of the laboratory frame, respectively. (b) $\left(\phi_{C}, \theta_{C}, \psi_{C}\right)$ are the rotation angles of the nanocrystal defined in the laboratory frame. The semi-cone angle $\Omega$ of signal collection is defined in the PSM geometry (c) and in the SPIM geometry (d). 


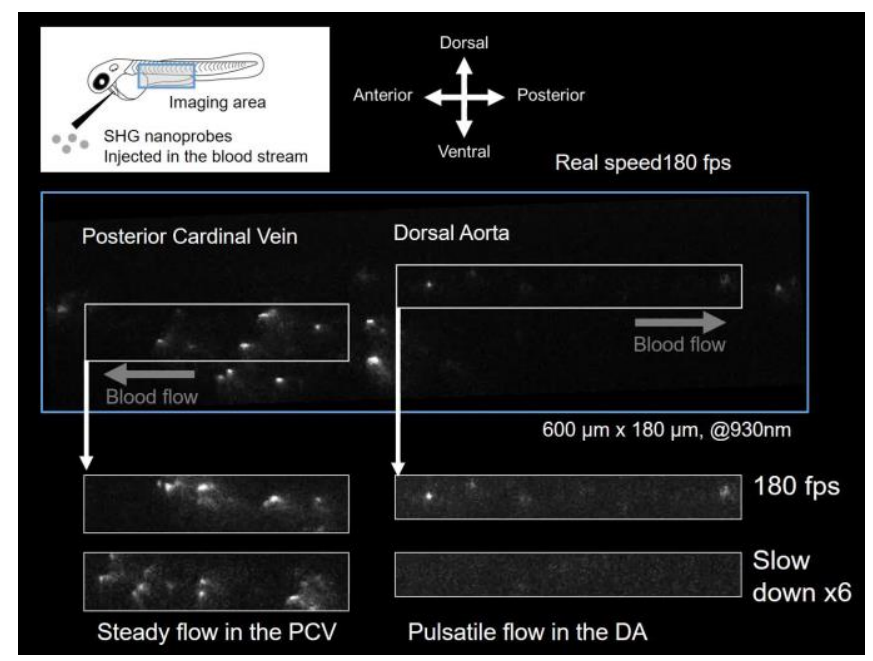

Movie 1. KTP nanocrystals imaged with SHG-SPIM at $180 \mathrm{fps}$ with $2 \mathrm{~ms}$ exposure time. A 3 dpf zebrafish embryo was injected with KTP nanocrystals and imaged at an excitation wavelength of $930 \mathrm{~nm}$. KTP nanocrystals flowing from right to left (rostral-to-caudal direction) can be seen in the posterior cardinal vein and flowing from left to right in the dorsal aorta. An area of $600 \times 180$ $\mu \mathrm{m}^{2}$ was imaged at $180 \mathrm{fps}$, corresponding to $>43 \mathrm{MHz}$ pixel rate.

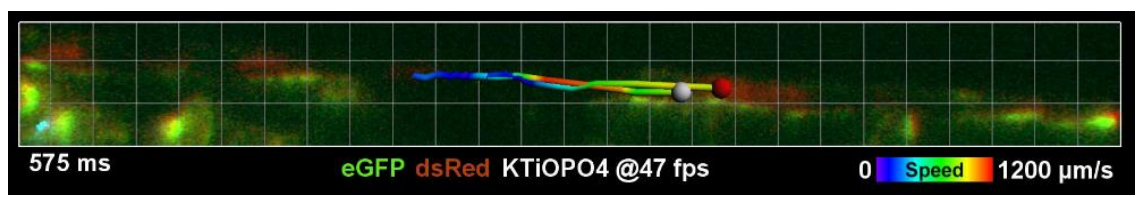

Movie 2. Multimodal in vivo imaging combining red and green fluorescence with SHG from nanoprobes. A 2.5dpf zebrafish embryo expressing eGFP in its endothelial cells and dsRed in its red blood cells (RBCs) was injected with KTP nanocrystals in the blood stream and imaged at 47 fps at an illumination wavelength of $930 \mathrm{~nm}$. The image sequence was taken in the trunk region and the fast pulsatile movement of RBCs and KTP nanocrystals flowing from left to right (caudalto-rostral direction) can be seen in the main artery. Simultaneous tracking a red blood cell (red dot) and a SHG nanoprobe (gray dot) moving at different speed and up to $1200 \mu \mathrm{m} / \mathrm{s}$. Grid scale $20 \mu \mathrm{m}$. 


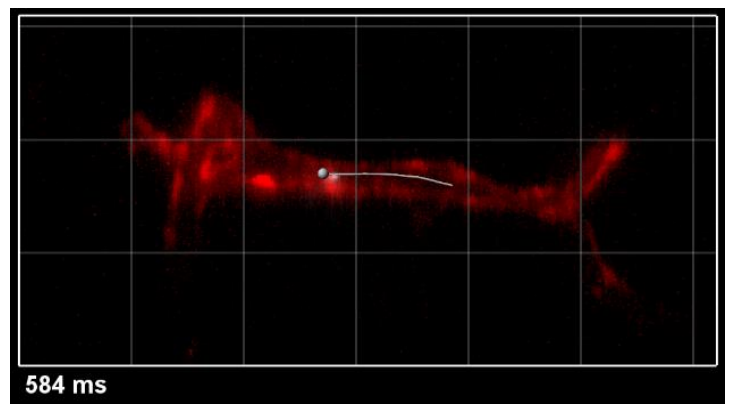

Movie 3. Multimodal in vivo imaging combining red fluorescence with SHG from nanoprobes. A $2.5 \mathrm{dpf}$ zebrafish embryo expressing mCherry in its endothelial cells was injected with KTP nanocrystals in the blood stream, and imaged at $65 \mathrm{fps}$ at an illumination wavelength of $1060 \mathrm{~nm}$. The movie was taken in the brain and show a nanocrystal flowing from the left mesencephalic vein into the left middle cerebral vein. This nanocrystal was tracked (gray dot and track) during $1.7 \mathrm{~s}$. At the end, 8 tracks are displayed (30s acquisition) showing nanocrystals flowing from the left or right mesencephalic vein into the left or right middle cerebral vein. Grid scale $50 \mu \mathrm{m}$.

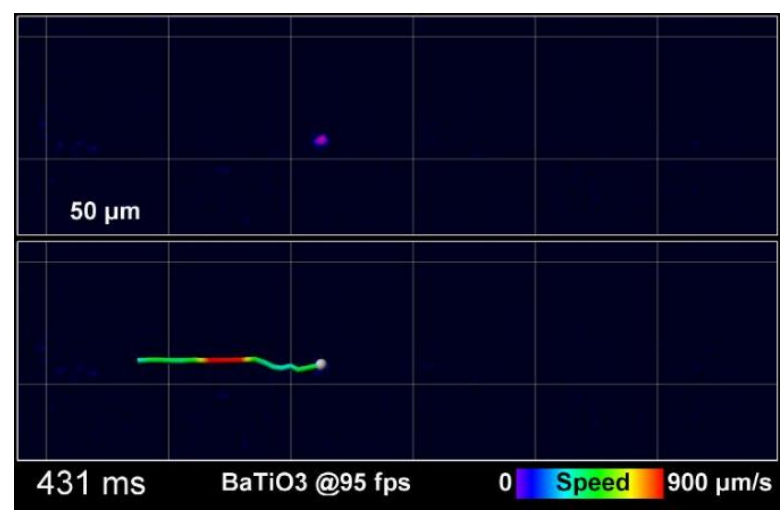

Movie 4. BT nanocrystals imaged with SHG-SPIM at 95 fps with $5 \mathrm{~ms}$ exposure time. A 3 dpf zebrafish embryo was injected with BT nanocrystals and imaged at an excitation wavelength of $930 \mathrm{~nm}$. BT nanocrystals flowing from left to right (caudal-to-rostral direction) can be seen in the main vein. Raw images (top) and tracked data (bottom). Color code of the trajectory code for instantaneous speed from 0 (blue) to $900 \mu \mathrm{m} / \mathrm{s}$ (red). Scale grid $50 \mu \mathrm{m}$. 


\section{Supporting Result 1. Sensitivity to incident polarization of SHG nanoprobes with strongest} signals

The sensitivity of SHG signal to incident polarization was investigated in Figure 3. While the results from simulations and experiments were very similar in the BT case (plain gray and blue lines in Figure 3d), we observed a stronger anisotropy in the experiments than in the simulations in the case of KTP nanocrystals (plain gray and red lines in Figure 3b). To identify possible causes for that difference, we plotted the mean signal from the $20 \%$ strongest or weakest among the 1000 simulated nanoprobes (doted and dashed gray lines in Figure 3, respectively). We note here that since all simulated particles have the same size, the level of detected SHG signals depends only on the $\chi^{(2)}$ tensors (i.e. the nature of the nanocrystals) and on the difference in relative orientation between the nanocrystal and the incident polarization. Interestingly, these two populations, i.e. strongest and weakest signals, exhibited a very different sensitivity to incident polarization, especially in the KTP case (Figure 3b and 3d). Indeed, the KTP nanocrystals with strongest detected signals exhibited a pronounced anisotropy, while the weakest ones did not $(0.11$ and 0.90 anisotropy factor, respectively). This calculation suggests that the observed difference between experiments and simulations in the KTP case could result from an experimental bias towards measuring mainly nanoprobes with the strongest signals. The difference in sensitivity to incident polarization between weak and strong images of nanoprobes is not as pronounced in the BT case and shows the opposite trend: the BT nanocrystals with strongest detected signals exhibited a lower anisotropy than the weakest ones ( 0.68 and 0.37 anisotropy factor, respectively).

\section{Supporting Result 2. Detection numerical aperture has effect on both mean and spread of SHG-SPIM signals}

To mitigate the spread of SHG signal intensities, we simulated the effect of detection NA ranging from 0.8 to 1.33 in SPIM using KTP or BT nanocrystals with both V-and H-polarization (Figure S3). We note that a NA of 1.33 corresponds to a collection of the total radiated SHG from randomly oriented nanoprobes when using a water immersion objective. Our experimental setup used a 0.8 NA detection objective, which can hardly be increased above 1 for practical reasons. As previously reported in the case of BT nanocrystals in PSM ${ }^{39}$, we found that increasing the detection NA in SPIM resulted in both an increase in signal mean level and a decrease in relative standard deviation when using a V-polarization, which is beneficial for imaging purposes (Figure S3). However, we 


\section{SUPPORTING INFORMATION}

noted that the reduction of signal spread was more pronounced for BT than for KTP (comparing Figure S3a and S3b, left). When using an H-polarization, KTP nanocrystals exhibited a specific behavior: despite a significant decrease in signal mean level, using a low detection NA could result in a lower signal spread (from 86 to $47 \%$, Figure S3a, right). In general, SPIM imaging of SHG nanoprobes can be improved by adjusting the detection NA, which has effect on both the signal mean level and its spread as a function of nanoprobe orientation. 